\title{
Coupling influences of nonlocal stress and strain gradients on dynamic pull-in of functionally graded nanotubes reinforced nano-actuator with damping effects
}

\author{
W. D. Yang, F. P. Yang and X. Wang* \\ School of Naval Architecture, Ocean and Civil Engineering \\ (State Key Laboratory of Ocean Engineering) \\ Shanghai Jiao Tong University, Shanghai 200240, P. R. China
}

\begin{abstract}
The paper reports a result of investigation on the dynamic pull-in instability of functionally graded carbon nanotubes (FGCNTs) reinforced nano-actuator considering damping behavior. Here, the nonlocal stress gradient and strain gradient theories are jointly utilized to capture the size effects of nanoscale structures. The material properties of FGCNTs reinforced nano-actuator are temperature-dependent, and the influences of geometrical nonlinearity and intermolecular forces such as van der Waals interaction and Casimir force are also considered. The results indicate that the fundamental frequency decreases with the increase of initial amplitude and electrostatic voltage until drops to zero when the dynamic pull-in occurs. It is shown that the frequencies of nano-actuator decline with the increment of nonlocal stress gradient parameter whereas increasing the strain gradient parameters enlarges the frequencies. In addition, it is observed from the time history and phase portrait that the dynamic pull-in voltage of system with damping behavior is larger than that without damping system, since the dissipative effect of damping requires more energy injected into the system.
\end{abstract}

Keywords: dynamic pull-in, carbon nanotube reinforced nano-actutor, nonlocal stress gradient, nonlocal strain gradient, Casimir force

\footnotetext{
* Corresponding author: X.Wang; E-mail address: xwang@sjtu.edu.cn (X.Wang).

Tel./fax: 086-021-54745367/086-021-54745367
} 


\section{Introduction}

Electrostatically actuated nano-device is widely applied in nano-switches [1], nano-tweezers [2] and nano-gyroscopes [3]. However, the dynamic pull-in phenomenon as one critical instability might lead the nano-actuators to fail, such as stiction, adhesion, and breakdown [4]. Therefore, investigations on dynamic pull-in instability of electrostatic nano-actuators have been reported in recent years.

With the continuing decrease of devices' characteristic size, some new phenomenon at nanoscale becomes significant, such as van der Waals interaction and Casimir force. Van der Waals force as the essential interaction among molecules, atoms and induced dipole particles, becomes dominant when the initial gap of two electrodes is less than characteristic wave length of material $[5,6]$. When the separation of macroscopic bodies is larger than $20 \mathrm{~nm}$, the interaction of multiple dipole particles within two macroscopic solids incorporating retarded effect is described as Casimir force [7-9]. For the electrostatic devices based on MEMS and NEMS(micro-electro-mechanical system and nano-electro-mechanical system), van der Waals interaction and Casimir force should be considered in the study of dynamic pull-in instability of nano-actuators.

Experimental observations indicate the size-dependent properties of nanoscale materials and structures, but the conventional continuum mechanics have no ability to embody the specific feature of size dependency. In recent years, some non-classical elasticity theories considering the effects of material length scales were proposed to investigate the size-dependent mechanical properties of sub-micron and nanoscale structures. These nonlocal elasticity theories are divided into two categories: nonlocal stress gradient model, for instance, Eringen's nonlocal stress gradient theory $[10,11]$, and nonlocal strain gradient model, such as (modified) couple stress theory [12-14] and strain gradient theory $[15,16]$.

Within the domain of pull-in instability of electrostatic devices, based on the nonlocal stress gradient theory, some investigations on the size-dependent electromechanical coupling influences of nano-actuators are presented in literatures 
[16-24]. From the observation of results, nonlocal stress gradient theory can well explain the softening stiffness with the increment of characteristic length parameter, but the main contribution of strain gradient is the stiffening of material $[13,16,17]$. Many works indicated the effects of stiffness softening by nonlocal stress gradient model and stiffness enhancement by nonlocal strain gradient model as the internal length scale parameters increase, respectively [22-25]. Thus, the aim of this work is to bring both of length scale parameters together into one unified constitutive equation to investigate the size effect of dynamic pull-in instability of nano-actuators. The exhibiting mechanical responses depend on the coupling influences of nonlocal stress gradient and strain gradient parameters for a specific materials and structures at nanoscale.

Moreover, functionally graded materials (FGMs) incorporating two or more constituent phases vary spatially in terms of non-uniform distribution of the reinforcement phase $[26,27]$. Due to the high strength ratio and high stiffness ratio, carbon nanotubes (CNTs) as one kind of promising reinforcement have been widely utilized in the field of nano-composite structures. There have been literatures that studied the nonlinear bending[28, 29], free vibration [30], buckling [31], thermal bucking and post-buckling [32-34] problems of functionally graded CNTs (FGCNTs) reinforced composites.

Furthermore, energy dissipation mechanism of electromechanical structure element plays a critical role in the performance of nano-actuators. Ghayesh et al. [35] investigated the effects of viscous damping and length-scale parameter on nonlinear dynamic behaviors of electrically actuated MEMS resonator based on modified couple stress theory. Azizi et al. [36] studied the thermoelastic damping of transversal vibration in a functionally graded piezoelectric microbeam resonator. And Liu and Wang [37] used hybrid differential transformation method to analyze the squeeze-film damping effect on nonlinear dynamic responses of microbeam actuator. Squeeze-film damping as a main source of dissipation in MEMS/NEMS are extrinsic damping, which is equivalently considered as viscous damping [38]. In addition, Tajaddodianfar et al. [39] investigated viscous and structural damping effects on size-dependent 
nonlinear dynamics of shallow nano-arch actuated by electrostatic force.

The present work focuses on the dynamic pull-in instability of FGCNTs reinforced nano-actuator with damping effects, and investigates the coupling effects of nonlocal stress gradient and strain gradient parameters by using the homotopy perturbation method (HPM). Results show that the damping effects (viscous and structural damping) lead to the systematical energy dissipation, thus alter the dynamic characteristics of nano-actuator subjected to electrostatic loads and intermolecular forces. In addition, the influences of temperature-dependent material properties and geometrical distribution of CNTs on fundamental frequency and dynamic pull-in are also described and discussed. Finally, the time story and phase portrait with damping effects are presented to analyze the coupling impacts of nonlocal stress gradient and strain gradient on the nonlinear dynamics characteristics of the size-dependent thermo-electro-mechanical system.

\section{Theoretical model and formulae}

Fig.1 shows a typical FGCNTs reinforced nano-actuator subjected to electrostatic force and dispersion force, such as van der Waals and Casimir force. The FGCNTs reinforced nano-actuator is considered as the long and thin nanobeam with the length $L$ and width $b$, where the thickness is $h_{e}$. The initial gap between the electrodes is $g$. The coordinate system is located to the neutral axis at the middle-left end of FGCNTs reinforced nano-actuator, where $x$ and $z$ present the horizontal and perpendicular direction, respectively.

\subsection{Constitutive relation based on unified stress and strain gradients}

Generally, the mechanical behavior of nano-materials appears in the characteristics of size-dependency, thus the investigation on size effect of nano-structure is important. Based on the Eringen's nonlocal stress gradient theory, the stress at a reference point in a body not only depends on the strain of this point, but also is influenced by all 
other points in the body, thus the nonlocal internal energy density potential is expressed as [18]

$$
U_{0}\left(\varepsilon_{i j}, \varepsilon^{\prime}{ }_{i j}, \alpha_{0}\right)=\frac{1}{2} \varepsilon_{i j} C_{i j k l} \int_{V} \alpha_{0}\left(\left|x-x^{\prime}\right|, e_{0} a\right) \varepsilon^{\prime}{ }_{k l} d V
$$

where $\varepsilon_{i j}$ and $\varepsilon_{i j}^{\prime}$ represent the Cartesian components of the strain tensors at points $x$ and $x^{\prime}$ within the domain $V ; C_{i j k l}$ is the elastic modulus tensor of classic elasticity and $\alpha_{0}$ is the attenuation kernel function referred to scale effect with Euclidean distance between two points; $e_{0}$ and $a$ are the nonlocal material constant and internal characteristic length, respectively.

To overcome the shortcoming of stiffness softening of nonlocal elasticity different from the experimental observation of nanoscale material, Lim et ale established the unified stress and strain gradient theory by incorporating nonlocal elasticity and strain gradient theory from two different physical characteristics of nanoscale material and structure [18]. Except for nonlocal effects of the classical strain field, the influence of higher-order strain gradients is considered. The extended internal energy density potential is described as [18]

$$
\begin{aligned}
U_{0}\left(\varepsilon_{i j}, \varepsilon_{i j}^{\prime}, \alpha_{0} ; \varepsilon_{i j, m}, \varepsilon_{i j, m}^{\prime}, \alpha_{1}\right)= & \frac{1}{2} \varepsilon_{i j} C_{i j k l} \int_{V} \alpha_{0}\left(\left|x-x^{\prime}\right|, e_{0} a\right) \varepsilon_{k l}^{\prime} d V \\
& +\frac{1}{2} l^{2} \varepsilon_{i j, m} C_{i j k l} \int_{V} \alpha_{1}\left(\left|x-x^{\prime}\right|, e_{1} a\right) \varepsilon_{k l, m}^{\prime} d V
\end{aligned}
$$

where $\varepsilon_{i j, m}$ and $\varepsilon_{i j, m}^{\prime}$ are higher-order counterparts of strain tensors at point $x$ and $x^{\prime} ; \alpha_{1}$ is another attenuation kernel function representing the nonlocal effect of the first-order strain gradient field; $l$ and $e_{1}$ are the material length scale originating from higher-order strain gradient field and related material constant, respectively.

If the improved higher-order nonlocal strain gradient theory is further simplified by assuming $e_{1}=e_{0}$ and keeping related terms of order $O\left(\nabla^{2}\right)$, the reduced model incorporating nonlocal elasticity and strain gradients is expressed as

$$
\left[1-\left(e_{0} a\right)^{2} \nabla^{2}\right] \sigma_{x x}=E_{e f f}\left(1-l^{2} \nabla^{2}\right) \varepsilon_{x x}
$$


where $E_{\text {eff }}$ represents the effective bulk modulus of solids. Note that the nonlocal elastic stress field can be obtained by setting material length scale $l=0$ as

$$
\left[1-\left(e_{0} a\right)^{2} \nabla^{2}\right] \sigma_{x x}=E_{e f f} \varepsilon_{x x}
$$

And the pure strain gradient constitutive is given by setting nonlocal material constant $e_{0}=0$ as

$$
\sigma_{x x}=E_{e f f}\left(1-l^{2} \nabla^{2}\right) \varepsilon_{x x}
$$

\subsection{Material properties of FGCNTs reinforced composite}

According to the extensive rule of mixture [40] and considering that material properties of CNTs and silicon are temperature-dependent, the effective material properties of CNTs reinforced composite can be expressed

$$
\begin{aligned}
& E_{\text {eff }}(z, T)=\eta V_{c n t}(z) E_{c n t}(T)+V_{m}(z) E_{m}(T) \\
& \alpha_{e f f}(z, T)=V_{c n t}(z) \alpha_{c n t}(T)+V_{m}(z) \alpha_{m}(T) \\
& \rho_{\text {eff }}(z, T)=V_{c n t}(z) \rho_{c n t}(T)+V_{m}(z) \rho_{m}(T)
\end{aligned}
$$

where $E_{\text {eff }}, \alpha_{\text {eff }}$ and $\rho_{\text {eff }}$ are the effective Young's modulus, thermal expansion

coefficient and mass density of CNTs reinforced composite, $E_{c n t}, \alpha_{c n t}$ and $\rho_{c n t}$ are the Young's modulus, thermal expansion coefficient and mass density of CNTs reinforcement, and $E_{m}, \alpha_{m}$ and $\rho_{m}$ are the Young's modulus, thermal expansion coefficient and mass density of matrix, respectively. $\eta$ is the efficiency parameter of CNTs induced by the size-dependent property of CNTs, and $V_{c n t}$ and $V_{m}$ are the volume fractions of CNTs and matrix.

Here, the distribution of CNTs is either uniformly distributed (UD-CNTRC) or functionally graded in thickness direction of the reinforced elastic layer, such as O-type (FGO-CNTRC) and X-type (FGX-CNTRC), shown as in Fig.1 [41]. All the geometrical distribution of CNTs considered in this work are expressed as 


$$
\begin{array}{ll}
V_{c n t}(z)=V_{c n t}^{*}, & (\mathrm{UD}-\mathrm{CNTRC}) \\
V_{c n t}(z)=2\left(1-2|z| / h_{e}\right) V_{c n t}^{*}, & (\mathrm{FGO}-\mathrm{CNTRC}) \\
V_{c n t}(z)=2\left(2|z| / h_{e}\right) V_{c n t}^{*}, & (\mathrm{FGX}-\mathrm{CNTRC})
\end{array}
$$

where

$$
V_{c n t}^{*}=\frac{m_{c n t}}{m_{c n t}+\rho_{c n t} / \rho_{m}-\left(\rho_{c n t} / \rho_{m}\right) m_{c n t}}
$$

and $m_{c n t}$ is the mass fraction of CNTs reinforcement. Therefore, the effective material properties of FGCNTs reinforced composite, such as $E_{\text {eff }}, \alpha_{\text {eff }}$ and $\rho_{\text {eff }}$, depend on environmental temperature and distribution type of CNTs reinforcement.

\subsection{Governing equation of FGCNTs reinforced nano-actuator}

Based on Euler-Bernoulli beam theory, the displacement at any point of the FGCNTs reinforced nano-actuator is defined as

$$
u_{x}(x, z, t)=u(x, t)-z \frac{\partial w(x, t)}{\partial x}, u_{y}(x, z, t)=0, u_{z}(x, z, t)=w(x, t)
$$

Where $u(x, t)$ and $w(x, t)$ are the displacement components of the mid-plane at time $t$. And the von Kármán type strain-displacement relation is expressed as

$$
\varepsilon_{x x}=\frac{\partial u_{x}}{\partial x}+\frac{1}{2}\left(\frac{\partial u_{z}}{\partial x}\right)^{2}=\frac{\partial u}{\partial x}-z \frac{\partial^{2} w}{\partial x^{2}}+\frac{1}{2}\left(\frac{\partial w}{\partial x}\right)^{2}
$$

Assuming that the FGCNTs reinforced nano-actuator is subjected to a thermal loading (the change of environmental temperature), the additional strains induced by the thermal loading are considered. Based on coupling nonlocal stress gradient and strain gradient theories [18], the constitutive relation of FGCNTs reinforced nano-actuator is defined as

$$
\left[1-\left(e_{0} a\right)^{2} \nabla^{2}\right] \sigma_{x x}(x, z)=E_{e f f}(z, T)\left(1-l^{2} \nabla^{2}\right)\left[\frac{\partial u}{\partial x}-z \frac{\partial^{2} w}{\partial x^{2}}+\frac{1}{2}\left(\frac{\partial w}{\partial x}\right)^{2}-\alpha_{e f f}(z, T) \Delta T\right]
$$

On the other hand, the axial force $N_{x}$ and bending moment $M_{x}$ induced by the 
stress fields are

$$
\begin{aligned}
& N_{x}=\int_{-1 / 2 h_{e}}^{1 / 2 h_{e}} \sigma_{x x}(x, z) b d z \\
& M_{x}=\int_{-1 / 2 h_{e}}^{1 / 2 h_{e}} \sigma_{x x}(x, z) z b d z
\end{aligned}
$$

Substituting Eq. (11) into Eqs. (12) and (13) leads to

$$
\begin{aligned}
& {\left[1-\left(e_{0} a\right)^{2} \frac{\partial^{2}}{\partial x^{2}}\right] N_{x}=\tilde{A}\left(1-l^{2} \frac{\partial^{2}}{\partial x^{2}}\right)\left[\frac{\partial u}{\partial x}+\frac{1}{2}\left(\frac{\partial w}{\partial x}\right)^{2}\right]-N^{T}} \\
& {\left[1-\left(e_{0} a\right)^{2} \frac{\partial^{2}}{\partial x^{2}}\right] M_{x}=-\tilde{D}\left(1-l^{2} \frac{\partial^{2}}{\partial x^{2}}\right) \frac{\partial^{2} w}{\partial x^{2}}-M^{T}}
\end{aligned}
$$

where $\{\tilde{A}, \tilde{D}\}=\int_{-1 / 2 h_{e}}^{1 / 2 h_{e}} E_{\text {eff }} b\left\{1, z^{2}\right\} d z$ are the stretching stiffness and bending stiffness, respectively, and $N^{T}$ and $M^{T}$ are the axial membrane force and bending moment induced by the thermal effect, as follows:

$$
N^{T}=\int_{-1 / 2 h_{e}}^{1 / 2 h_{e}} E_{e f f}(z, T) \alpha_{e f f}(z, T) \Delta T b d z, M^{T}=\int_{-1 / 2 h_{e}}^{1 / 2 h_{e}} E_{e f f}(z, T) \alpha_{e f f}(z, T) \Delta T z b d z
$$

By employing Hamilton's principle and no-considering the external axial force, the dynamic equilibrium equations of electrical actuator with damping systems are expressed as

$$
\begin{gathered}
\frac{\partial N_{x}}{\partial x}=I_{1} \frac{\partial^{2} u}{\partial t^{2}} \\
\frac{\partial^{2} M_{x}}{\partial x^{2}}+\frac{\partial}{\partial x}\left(N_{x} \frac{\partial w}{\partial x}\right)+f_{z}-C_{v d} \frac{\partial w}{\partial t}-C_{s d} \frac{\partial^{2} w}{\partial t \partial x}=I_{1} \frac{\partial^{2} w}{\partial t^{2}}
\end{gathered}
$$

where $I_{1}=\int_{-1 / 2 h_{e}}^{1 / 2 h_{e}} \rho_{e f f}(z, T) b d z$ is the inertia of nano-actuator.

In Eq.(16b), $C_{v d}$ and $C_{s d}$ are, respectively, defined as the viscous and structural damping coefficients based on a generalized Kelvin model, in which the viscous damping rises from the transverse deformation velocity of beam and the structural damping rises from the rotation velocity of the cross section of beam [39].

In the above formula, $f_{z}$ is the transversely distributed force which is composed of the electrostatic force with first-order corrected fringing field effect, van der Waals force per unit length and Casimir force, as follows: 


$$
f_{z}=F_{e}+F_{v d W}+F_{C a s}
$$

where, $F_{e}$ is the electrostatic force per unit length exerted on the nano-actuator as follows [42]

$$
F_{e}=\frac{\varepsilon_{0} \bar{V}_{e}^{2} b}{2(g-w)^{2}}\left(1+0.65 \frac{g-w}{b}\right)
$$

here $\varepsilon_{0}=8.854 \times 10^{-12} C^{2} N^{-1} m^{-2}$ is the permittivity of vacuum, $\bar{V}_{e}$ is the applied electrostatic voltage and $g$ is the initial gap between the bottom surface of nano-device and the substrate.

In Eq.(17), $F_{v d W}$ is van der Waals force per unit length induced by the intermolecular attraction between nano-actuator and the substrate, as follows[43]

$$
F_{v d W}=\frac{\bar{A} b}{6 \pi(g-w)^{3}}
$$

where $\bar{A}$ is the Hamaker constant.

In Eq.(17), $F_{\text {Cas }}$ is the Casimir force as the macro effect of quantum field fluctuation per unit length, as follows [44]

$$
F_{\text {Cas }}=\frac{\pi^{2} \mathrm{~h} c_{0} b}{240(g-w)^{4}}
$$

where $\mathrm{h}=1.055 \times 10^{-34} J s$ is the Planck's constant divided by $2 \pi$ and $c=3 \times 10^{8} \mathrm{~m} \cdot \mathrm{s}^{-1}$ is the speed of light.

Substituting Eqs. (14a) and (14b) into Eq. (16a) and neglecting the axial accelerator of nano-actuator give

$$
\begin{aligned}
& \frac{\partial N_{x}}{\partial x}=0 \Leftrightarrow \tilde{A}\left[\frac{\partial u}{\partial x}+\frac{1}{2}\left(\frac{\partial w}{\partial x}\right)^{2}\right]=C_{1}(t) \\
& \Rightarrow \tilde{A}\left[u+\int_{0}^{x} \frac{1}{2}\left(\frac{\partial w}{\partial x}\right)^{2} d x\right]=x C_{1}(t)+C_{2}(t)
\end{aligned}
$$

where $C_{1}$ and $C_{2}$ are integration of constant based on time. After some 
mathematical manipulations, one has $C_{1}(t)=\frac{\tilde{A}}{L} \int_{0}^{L} \frac{1}{2}\left(\frac{\partial w}{\partial x}\right)^{2} d x$ and $C_{2}(t)=0$. Thus, the term of axial force is summarized to

$$
N_{x}=\frac{\tilde{A}}{L} \int_{0}^{L} \frac{1}{2}\left(\frac{\partial w}{\partial x}\right)^{2} d x-N^{T}
$$

Substituting Eqs.(14b) and (22) into Eq.(16b), yields

$$
\begin{aligned}
M_{x} & =\left(e_{0} a\right)^{2}\left[I_{1} \frac{\partial^{2} w}{\partial t^{2}}-\frac{\partial}{\partial x}\left(N_{x} \frac{\partial w}{\partial x}\right)-f_{z}+C_{v d} \frac{\partial w}{\partial t}\right. \\
& \left.+C_{s d} \frac{\partial^{2} w}{\partial t \partial x}\right]-\tilde{D}\left(1-l^{2} \frac{\partial^{2}}{\partial x^{2}}\right) \frac{\partial^{2} w}{\partial x^{2}}-M^{T} \\
& =\left(e_{0} a\right)^{2}\left\{I_{1} \frac{\partial^{2} w}{\partial t^{2}}-\left[\frac{\tilde{A}}{L} \int_{0}^{L} \frac{1}{2}\left(\frac{\partial w}{\partial x}\right)^{2} d x-N^{T}\right] \frac{\partial^{2} w}{\partial x^{2}}-f_{z}\right. \\
& \left.+C_{v d} \frac{\partial w}{\partial t}+C_{s d} \frac{\partial^{2} w}{\partial t \partial x}\right\}-\tilde{D}\left(1-l^{2} \frac{\partial^{2}}{\partial x^{2}}\right) \frac{\partial^{2} w}{\partial x^{2}}-M^{T}
\end{aligned}
$$

Then substituting Eq.(22) and Eq.(23) into Eq.(14b) generates the governing equation based on nonlocal elasticity and strain gradients, as follows:

$$
\begin{gathered}
\left\{\tilde{D}+\left(e_{0} a\right)^{2}\left[\frac{\tilde{A}}{L} \int_{0}^{L} \frac{1}{2}\left(\frac{\partial w}{\partial x}\right)^{2} d x-N^{T}\right]\right\} \frac{\partial^{4} w}{\partial x^{4}}-\tilde{D} l^{2} \frac{\partial^{6} w}{\partial x^{6}}+I_{1} \frac{\partial^{2} w}{\partial t^{2}} \\
-\left(e_{0} a\right)^{2} I_{1} \frac{\partial^{4} w}{\partial t^{2} \partial x^{2}}-\left[\frac{\tilde{A}}{L} \int_{0}^{L} \frac{1}{2}\left(\frac{\partial w}{\partial x}\right)^{2} d x-N^{T}\right] \frac{\partial^{2} w}{\partial x^{2}} \\
-\left[1-\left(e_{0} a\right)^{2} \frac{\partial^{2}}{\partial x^{2}}\right]\left(f_{z}-C_{v d} \frac{\partial w}{\partial t}-C_{s d} \frac{\partial^{2} w}{\partial t \partial x}\right)=0
\end{gathered}
$$

where the nonlocal governing equation can be obtained by setting $l=0$ and the pure strain gradient governing equation can be given by setting $e_{0}=0$.

Substituting the transversely distributed forces $f_{z}$ in Eq.(17) which represents the sum of all dispersion forces from different physical origins, including electrostatic force incorporating fringing field effect $f_{\text {elec }}$, van der Waals force $f_{v d W}(n=0)$ and Casimir force $f_{\text {Cas }}(n=1)$ into Eq. (24) leads to the dimensionless governing equation based on nonlocal elasticity and strain gradients, as follows: 


$$
\begin{aligned}
& K_{3} \frac{\partial^{4} W}{\partial \xi^{4}}+\mu\left[K_{1} \int_{0}^{1}\left(\frac{\partial W}{\partial \xi}\right)^{2} d \xi-K_{2} N^{T}\right] \frac{\partial^{4} W}{\partial \xi^{4}}-\eta K_{3} \frac{\partial^{6} W}{\partial \xi^{6}}+K_{4} \frac{\partial^{2} W}{\partial \tau^{2}}-\mu K_{4} \frac{\partial^{4} W}{\partial \xi^{2} \partial \tau^{2}} \\
& -\left[K_{1} \int_{0}^{1}\left(\frac{\partial W}{\partial \xi}\right)^{2} d \xi-K_{2} N^{T}\right] \frac{\partial^{2} W}{\partial \xi^{2}}+\tilde{C}_{s d}\left(\frac{\partial^{2} W}{\partial \tau \partial \xi}-\mu \frac{\partial^{4} W}{\partial \tau \partial \xi^{3}}\right)+\tilde{C}_{v d}\left(\frac{\partial W}{\partial \tau}-\mu \frac{\partial^{3} W}{\partial \tau \partial \xi^{2}}\right) \\
& -\left(1-\mu \frac{\partial^{2}}{\partial \xi^{2}}\right)\left[\frac{V_{e}^{2} \gamma}{1-W}+\frac{V_{e}^{2}}{(1-W)^{2}}+\frac{(1-n) \alpha_{3}}{(1-W)^{3}}+\frac{n \alpha_{4}}{(1-W)^{4}}\right]=0
\end{aligned}
$$

In the above formula, the dimensionless variables are given by

$$
\begin{gathered}
\xi=\frac{x}{L}, W=\frac{w}{g}, \tau=t \sqrt{\frac{D_{m}}{I_{m} L^{4}}} \\
K_{1}=\frac{\tilde{A} g^{2}}{2 D_{m}}, K_{2}=\frac{L^{2}}{D_{m}}, K_{3}=\frac{\tilde{D}}{D_{m}}, \quad K_{4}=\frac{I_{1}}{I_{m}}, \\
\mu=\left(\frac{e_{0} a}{L}\right)^{2}, \eta=\left(\frac{l}{L}\right)^{2}, \tilde{C}_{s d}=\frac{L}{\sqrt{D_{m} I_{m}}} C_{s d}, \tilde{C}_{v d}=\frac{L^{2}}{\sqrt{D_{m} I_{m}}} C_{v d}, \\
V_{e}=\bar{V}_{e} \sqrt{\frac{\varepsilon_{0} L^{4}}{2 g^{3} D_{m}}}, \gamma=0.65 \frac{g}{b}, \alpha_{3}=\frac{\bar{A} L^{4}}{6 \pi g^{4} D_{m}}, \alpha_{4}=\frac{\pi^{2} \mathrm{~h} c_{0} L^{4}}{240 g^{5} D_{m}}
\end{gathered}
$$

where $D_{m}$ and $I_{m}$ represent the bending stiffness and moment of inertia of nano-actuator without CNTs reinforcement, respectively. In order to solve 6th order differential equation (25) based on nonlocal stress-strain gradients, for the clamped-clamped nano-actuator, four nondimensional classical boundary conditions and two additional unclassical boundary conditions originate from strain gradients are expressed as [45]:

$$
\begin{gathered}
\left.W(\xi, \tau)\right|_{\xi=0}=0,\left.W(\xi, \tau)\right|_{\xi=1}=0, \\
\left.\frac{\partial W(\xi, \tau)}{\partial \xi}\right|_{\xi=0}=0,\left.\quad \frac{\partial W(\xi, \tau)}{\partial \xi}\right|_{\xi=1}=0 \\
\left.\frac{\partial^{2} W(\xi, \tau)}{\partial \xi^{2}}\right|_{\xi=0}=0,\left.\frac{\partial^{2} W(\xi, \tau)}{\partial \xi^{2}}\right|_{\xi=1}=0
\end{gathered}
$$

For the nonlinear dynamic system, applying the Galekin's decomposition method to Eq. (25) yields to a second-order nonlinear ordinary differential equation [46]. Assume the deflection function has the following form as 


$$
W(\xi)=q(\tau) \phi(\xi)
$$

where $q(\tau)$ represents an undetermined time-dependent function and $\phi(\xi)$ is the linear mode shape function satisfying the boundary condition (27), which is given by

$$
\phi(\xi)=\cosh \lambda_{m} \xi-\cos \lambda_{m} \xi-\frac{\cosh \lambda_{m}-\cos \lambda_{m}}{\sinh \lambda_{m}-\sin \lambda_{m}}\left(\sinh \lambda_{m} \xi-\sin \lambda_{m} \xi\right)
$$

where $\lambda_{m}$ is the $m$-th root of characteristic equation of clamped-clamped nanobeam.

Substituting Eqs. (28) and (29) into Eq. (25), using Taylor's series expansion for dimensionless transversely distributed forces (i.e. four-order expansion of deflection having high enough accuracy), and multiplying by the linear mode shape function $\phi(\xi)$ and then integrating along the nano-actuator span, one can obtain

$$
\ddot{q}(\tau)+\psi_{5} \dot{q}(\tau)+\psi_{4}(q(\tau))^{4}+\psi_{3}(q(\tau))^{3}+\psi_{2}(q(\tau))^{2}+\psi_{1} q(\tau)+\psi_{0}=0
$$

where the parameters $\psi_{i}, i \in[0,5]$ are list in Appendix A.

\section{Closed-form equations of frequency and amplitude}

The capacitive-type MEMS and NEMS devices are generally actuated by a step voltage. When an external voltage is not applied, the system is at rest and has no stored energy; however, when a step voltage is applied, the energy of the system is stored as kinetic and potential energy. Over time, the stored energy before related to the equilibrium position is dissipated though damping effects (i.e. viscous and structural damping). According to energy balance of the system at any time, one obtains [47]

$$
E_{\text {system }}=E_{\text {kinetic }}+E_{\text {potential }}+E_{\text {dissipated }}
$$

where $E_{\text {system }}$ is the energy inputting into the system, $E_{\text {kinetic }}$ is the kinetic energy of the system, $E_{\text {potential }}$ is the elastic potential energy stored in the system and $E_{\text {dissipated }}$ is the dissipated energy from damping effects of the system.

To achieve the lowest value of the dynamic pull-in voltage, the damping effects are 
generally suppressed. The minimum dynamic pull-in voltage can be obtained by neglecting the damping effect, thus Eq. (30) can be rewritten to further calculate the dynamic pull-in voltage without damping effect, as follows:

$$
\ddot{q}(\tau)+\psi_{4}(q(\tau))^{4}+\psi_{3}(q(\tau))^{3}+\psi_{2}(q(\tau))^{2}+\psi_{1} q(\tau)+\psi_{0}=0
$$

The basic character of the HPM is to employ homotopy technique and embed a parameter which typically ranges from zero to one. When the embedding parameter is zero, the equation is one of a linear system; when it is one, the equation is the same as the original one[48].

To illustrate the basic idea of the HPM, consider the following nonlinear differential equation,

$$
\mathscr{A}(a)-f(r)=0, \quad r \in \Omega
$$

with associated boundary conditions as

$$
\mathscr{B}\left(a, \frac{\partial a}{\partial s}\right)=0, r \in \Gamma
$$

where $\mathscr{A}$ is a general differential operator, $\mathscr{B}$ is a boundary operator, $f(r)$ is a known analytical function, and $\Gamma$ is the boundary of the domain $\Omega$. In general, the operator $\mathscr{A}$ consists of linear part $\mathscr{L}$ and nonlinear $\mathscr{N}$. Thus, Eq. (33) is expressed as

$$
\mathscr{L}(a)+\mathscr{N}(a)-f(r)=0
$$

Based on homotopy technique, a homotopy is constructed as [49]

$$
\mathscr{H}(v, p)=(1-p)\left[\mathscr{L}(v)-\mathscr{L}\left(a_{0}\right)\right]+p[\mathscr{A}(a)-f(r)]=0
$$

where $p \in[0,1]$ denotes the embedding parameter and $a_{0}$ is an initial value for the solution of Eq. (33). Note that the homotopy $\mathscr{H}(v, p)$ depends on not only the undetermined variable $a$, but also the embedding parameter $p \in[0,1]$. The solution of Eq. (36) can be described as a power series of $p$ as

$$
v=v_{0}+p v_{1}+p^{2} v_{2}+p^{3} v_{3}+\mathrm{L}
$$


and the embedding parameter $p$ is used to expand the square of the undetermined oscillation fundamental frequency as

$$
\omega_{0}=\omega^{2}-p \omega_{1}-p^{2} \omega_{2}-\mathrm{L}
$$

where $\omega_{0}$ is the coefficient of $a(r)$ in Eq. (36), satisfying kinetic boundary conditions, and the coefficients $\omega_{j}(j=1,2, \ldots)$ are undetermined parameters.

When $p \rightarrow 1$, the approximated solution of Eq. (35) and the fundamental frequency $\omega$, respectively, are expressed as

$$
\begin{gathered}
a=\lim _{p \rightarrow 1} v=v_{0}+v_{1}+v_{2}+v_{3}+\mathrm{L} \\
\omega^{2}=\omega_{0}+\omega_{1}+\omega_{2}+\mathrm{L}
\end{gathered}
$$

Utilizing HPM to solve Eq. (32), the homotopy equation can be constructed in the following form as

$$
\mathscr{H}(q, p)=(1-p)\left[\ddot{q}+\psi_{1} q\right]+p\left[\ddot{q}+\psi_{0}+\psi_{1} q+\psi_{2} q^{2}+\psi_{3} q^{3}+\psi_{4} q^{4}\right]=0
$$

Suppose the solution of Eq.(32) has the following form as

$$
q(\tau)=q_{0}(\tau)+p q_{1}(\tau)+p^{2} q_{2}(\tau)+\mathrm{L}
$$

And the coefficient of $q$ is expanded into a series of $p$ as

$$
\psi_{1}=\omega^{2}-p \omega_{1}-p^{2} \omega_{2}-\mathrm{L}
$$

Substituting Eqs. (42)and (43) into Eq. (41), and equating the terms with the identical powers of $p$,

$$
\begin{aligned}
p^{0}: & (\tau)+\omega^{2} q_{0}(\tau)=0, \quad q_{0}(0)=A, \quad \&(0)=0, \\
p^{1}: & (\tau)+\omega^{2} q_{1}(\tau)=\omega_{1} q_{0}(\tau)-\left[\psi_{0}+\psi_{2}\left(q_{0}(\tau)\right)^{2}+\psi_{3}\left(q_{0}(\tau)\right)^{3}+\psi_{4}\left(q_{0}(\tau)\right)^{4}\right], \\
& q_{1}(0)=0, \quad \&(0)=0, \\
p^{2}: & (\tau)+\omega^{2} q_{2}(\tau)=\omega_{1} q_{1}(\tau)+\omega_{2} q_{0}(\tau)-\left[2 \psi_{2} q_{0}(\tau) q_{1}(\tau)+3 \psi_{3}\left(q_{0}(\tau)\right)^{2} q_{1}(\tau)\right. \\
& \left.+4 \psi_{4}\left(q_{0}(\tau)\right)^{3} q_{1}(\tau)\right], \quad q_{2}(0)=0, \quad \&(0)=0
\end{aligned}
$$

It is easy to obtain the solution of Eq. (44a) as 


$$
q_{0}(\tau)=A \cos (\omega \tau)
$$

Because the solution of Eq. (44b) should not incorporate the so-called secular term $\cos (\omega \tau), \quad$ substituting Eq.(45) into Eq. (44b) generates

$$
\begin{gathered}
+\omega^{2} q_{1}(\tau)=\left(\omega_{1} A-\frac{3}{4} \psi_{3} A^{3}\right) \cos (\omega \tau)-\left(\frac{1}{2} \psi_{2} A^{2}+\frac{1}{2} \psi_{4} A^{4}\right) \cos (2 \omega \tau) \\
-\frac{1}{2} \psi_{2} A^{2}-\frac{3}{8} \psi_{4} A^{4}-\psi_{0}-\frac{1}{4} \psi_{3} A^{3} \cos (3 \omega \tau)-\frac{1}{8} \psi_{4} A^{4} \cos (4 \omega \tau)
\end{gathered}
$$

Note the secular terms of Eq. (46) should be zero, thus one can obtain

$$
\omega_{1}=\frac{3}{4} \psi_{3} A^{2}
$$

Solving the updated Eq. (46) can give rise to one second-order approximated expression of $q_{1}(\tau)$ as

$$
\begin{aligned}
q_{1}(\tau)= & \frac{1}{480 \omega^{2}}\left[\left(96 \psi_{4} A^{4}+160 \psi_{2} A^{2}-15 \psi_{3} A^{3}+480 \psi_{0}\right) \cos (\omega \tau)\right. \\
& +\left(80 \psi_{4} A^{4}+80 \psi_{2} A^{2}\right) \cos (2 \omega \tau)+15 \psi_{3} A^{3} \cos (3 \omega \tau) \\
& \left.+4 \psi_{4} A^{4} \cos (4 \omega \tau)-\left(480 \psi_{0}+180 \psi_{4} A^{4}+240 \psi_{2} A^{2}\right)\right]
\end{aligned}
$$

Furthermore, Eq. (43) with two terms approximation of power series when $p \rightarrow 1$,

$$
\omega_{2}=\omega^{2}-\psi_{1}-\omega_{1}
$$

Substituting Eq. (49) into Eq. (44c) for $q_{2}(\tau)$ and requiring the secular terms to be zero lead to

$$
\begin{aligned}
S(\omega)= & -\omega^{4} A+\psi_{1} \omega^{2} A+\frac{3}{4} \psi_{3} \omega^{2} A^{3}-2 \psi_{0} \psi_{2} A-\frac{5}{6} \psi_{2}^{2} A^{3} \\
& +\frac{3}{2} \psi_{0} \psi_{3} A^{2}+\frac{1}{2} \psi_{2} \psi_{3} A^{4}-\frac{3}{128} \psi_{3}^{2} A^{5}-3 \psi_{0} \psi_{4} A^{3} \\
& -\frac{7}{4} \psi_{2} \psi_{4} A^{5}+\frac{3}{10} \psi_{3} \psi_{4} A^{6}-\frac{63}{80} \psi_{4}^{2} A^{7}=0
\end{aligned}
$$

By solving Eq. (50) respect to the fundamental frequency $\omega$, the analytical function of second-order frequency-amplitude relationship is obtained as 


$$
\begin{aligned}
\omega(A)= & {\left[\frac{1}{2} \psi_{1}+\frac{3}{8} \psi_{3} A^{2}+\left(\frac{1}{4} \psi_{1}^{2}-2 \psi_{0} \psi_{2}+\frac{3}{2} \psi_{0} \psi_{3} A+\frac{3}{8} \psi_{1} \psi_{3} A^{2}-3 \psi_{0} \psi_{4} A^{2}-\frac{5}{6} \psi_{2}^{2} A^{2}\right.\right.} \\
& \left.\left.+\frac{1}{2} \psi_{2} \psi_{3} A^{3}+\frac{15}{128} \psi_{3}^{2} A^{4}-\frac{7}{4} \psi_{2} \psi_{4} A^{4}+\frac{3}{10} \psi_{3} \psi_{4} A^{5}-\frac{63}{80} \psi_{4}^{2} A^{6}\right)^{1 / 2}\right]^{1 / 2}
\end{aligned}
$$

\section{Results and discussion}

\subsection{Validation of analytical method}

To check the validity and accuracy of the HPM used in the paper, the results calculated by the HPM are compared with the experimental data and numerical methods. Gupta et al [50] fabricated one doubly-clamped polysilicon microbeam and the parameters of the microbeam are listed in Table 1. The comparisons of dynamic pull-in voltage obtained from different methods are listed in Table 2. $\sigma_{0}$ is the gap-dependent residual stress induced by thermal mismatch and fabrication of micromachining. It is seen from Table 2 that the present results appear in good agreement with the experimental data, and the dynamic pull-in voltage calculated in present model is also well agree with those by other methods, such as Displacement Iteration Pull-In Extraction (DIPIE algorithm) [51] and Energy Balance Method (EBM)[52]. The accuracy of HPM in the paper is also verified by comparing with those by the Runge-Kutta method, shown as in Fig.2, where UD-CNTRC type nano-actuator with Casimir force is considered, and $V_{e}=8, V_{c n t}=0.12, \mu=0.01$, $\Delta T=200 K$ and $\alpha_{4}=10$. It can be seen from Fig.2 that the non-dimensional amplitudes by the second-order HPM have enough high accuracy with the results by numerical method. In addition, Table 3 describes the convergence of HPM when van der Waals force and Casimir force are, respectively, considered. The absolute error decreases with the increase of initial amplitude, but the highest error are only $0.48 \%$ for van der Waals force and $0.65 \%$ for Casimir force, respectively. Therefore the HPM used in the paper exhibits good accuracy and convergence in investigating dynamic 
pull-in of FGCNTs reinforced nano-actuator.

To further verify present analytical model, a comparison of the present method with nonlocal stress and strain gradient theory from references $[1,53,54]$ has been given in Table 4, in which the comparison shows that the present HPM has good accuracy to compute the pull-in voltage of nano-actuator with size-dependency.

\subsection{Coupling effects of stress and strain gradients}

Table 5 gives the temperature-dependent material properties of CNTs and silicon matrix under the temperature conditions of $300 \mathrm{~K}$ (room temperature), 400K, 500K, $600 \mathrm{~K}$ and $700 \mathrm{~K}[28,55,56]$, where we select the elastic modulus [1 $\overline{1} 0]$ direction silicon and perform data fitting to obtain the values for corresponding temperature points. The resultant temperature-dependent elastic modulus of silicon satisfy the relationship of $E_{m}=(175.639-0.01987 T) \mathrm{MPa} \quad, \quad$ where $T=T_{0}+\Delta T \quad$ and $T_{0}=300 \mathrm{~K}$ (room temperature). Table 6 lists the scale-dependent efficiency parameter of CNTs for volume fraction of $0.12,0.17$ and 0.28 which are determined by MD simulation and the rule of mixture incorporating scale-dependent effect [40]. The physical and geometrical parameters of FGCNTs reinforced nano-actuator for latter numerical analysis are taken as $h_{e}=10 \mathrm{~nm}, L=25 h_{e}, g=0.3 h_{e}$ and $b=4.5 h_{e}$, respectively.

The respective influences of nonlocal stress gradient and strain gradient on relation of fundamental frequency and applied voltage are shown in Fig.3. When the voltage increases to a certain critical value, the dynamic pull-in phenomenon happens; this critical voltage is called dynamic pull-in voltage. It is found that the frequency of system declines with the increase of applied voltage, and the frequency drops to zero at the pull-in state. Furthermore, the magnitude of frequency decreases with the increase of nonlocal parameter, but with the decrease of strain gradient parameter. Moreover, the influence of nonlocal stress field theory decreases the stiffness of nano-actuator as the increase of the nonlocal parameter, whereas the increase of the 
high-order strain gradient induces the stiffness of nano-actuator to increase.

Fig.4 depicts the influences of geometrical distribution of CNTs on the vibration frequency of nano-actuator for different nonlocal stress gradient and strain gradient models. It is shown that based on the nonlocal stress gradient model, the FGX-CNTRC nano-actuator undergoes the wider range of variation of frequency as the nonlocal parameter increases by comparing among the UD-CNTRC and FGO-CNTRC type nano-actuator. Based on the nonlocal strain gradient model, the effect of distribution form of CNTs in nano-actuator does not change the variable range of frequency of the nano-actuator.

Fig.5 demonstrates the effects of volume fraction of CNTs in nano-actuator on the frequency of the nano-actuator based on different nonlocal stress and strain gradient models. It is concluded that to enhance the volume fraction of CNTs leads to the increase of fundamental frequency of nano-actuator, since the increment of CNTs in nano-actuator improves the stiffness of the nano-actuator. Moreover, the frequency of nano-actuator declines with the increase of stress gradient parameter but increases with the increase of strain gradient parameter.

The influences of environmental temperature change on the frequency of nano-actuator are illustrated in Fig.6. It is seen that the effect of temperature change on the frequency of nano-actuator based on different nonlocal models are significant, and the increase of nonlocal stress gradient parameter decreases the frequency of nano-actuator for different temperature changes. On the other hand, the influence of strain gradient parameter on the differences of frequency at different temperature change is small, since the influence of nonlocal stress gradient is larger than that of higher-order strain gradient.

Fig.7 describes the effects of van der Waals force and Casimir force on the frequency of nano-actuator for two nonlocal models. It is seen that as the nonlocal stress gradient parameter increases the effects of van der Waals force and Casimir force on the frequency of nano-actuator gradually increase, but as the strain gradient parameter increases the effects of van der Waals force and Casimir force on the frequency of nano-actuator gradually decrease. Also, the frequency of nano-actutor 
decrease with the increment of van der Waals force and Casimir force until the pull-in instability occurs.

Fig.8 depicts the nonlinear coupling effects of nonlocal stress gradient and strain gradient on the fundamental frequency of nano-actuator. It is found that the influences of stress and strain gradient parameters on the frequency nano-actuator are contrary and the relation of frequency and stress/strain gradient parameters shows significantly nonlinear. If the material constant, characteristic length parameter and strain gradient parameter of nanoscale materials and structures are determined, the unified stress and strain gradient model is able to accurately predict the dynamic behaviors and dynamic pull-in instability of nano-actuator incorporating size effect.

Furthermore, the influences of stress gradient and strain gradient parameters on the frequencies of first three modes of nano-actuator have also been described. The first three frequencies as functions of applied voltage are discussed, and the new results are shown in Fig.9. It is seen from Fig.9 that the nonlocal stress gradient parameter significantly decreases the frequencies of modes before the pull-in voltage; whereas, the frequencies of modes increase as the strain gradient parameter increases. In addition, because the pull-in instability firstly occurs at the primary frequency, thus the frequencies of higher modes might still exist after pull-in state of nano-actuator, which is similar to the result from Ref.[57].

\subsection{Dynamic pull-in and bifurcation analysis}

To investigate the dynamic characteristics of nano-actuator near pull-in state, the curves of time history and phase portrait of the nano-actuator under different applied voltages are illustrated in Fig.10. The nonlocal stress and strain gradient parameters are taken as $\mu=0.1$ and $\eta=0.1$; the non-dimensional structural and viscous damping parameters are $\tilde{C}_{s d}=1$ and $\tilde{C}_{v d}=1$, respectively. It is from Fig.10(a) that when applied a lower voltage (i.e. $\mathrm{V}_{\mathrm{e}}=17.78$ for UD-CNTRC type nano-actuator), the non-dimension deflection decreases as the non-dimension time increases due to the structural and viscous damping effects; whereas if increasing the applied voltage to 
the dynamic pull-in value (i.e. $\mathrm{V}_{\mathrm{e}}=17.79$ ), the system becomes unstable and the nano-actuator collapses onto the substrate. Moreover, it is found from Fig.10(b) that before the pull-in state, the phase portrait of system has one stable center, and as the time history progresses, the phase portrait converges into the center point. However, the system becomes dynamically unstable at the pull-in state and appears bifurcation from the unstable saddle node. When the voltage beyond the pull-in voltage (i.e. $\mathrm{V}_{\mathrm{e}}=17.78$ ), the stable center vanishes and the nano-actuator sticks onto the substrate.

In order to observe the influences of damping effects, Fig.11 depicts the curves of time history and phase portrait of nano-actuator without damping effects ( $\tilde{C}_{s d}=0$ and $\tilde{C}_{v d}=0$ ). It is obviously seen that compared to the dynamic pull-in voltage with damping, the dynamic pull-in voltage of system without damping effects decreases to $\mathrm{V}_{\mathrm{e}}=17.72$ and the maximum velocity of system is slightly smaller, since the dissipation of energy injected into system increases the dynamic pull-in voltage of the damping nano-actuator. The non-dimensional deflection of nano-actuator without damping keep the identical amplitude, and the system without damping has one stable center and there exists periodic orbits around the center. When the value of voltage surpasses the pull-in voltage, the stable center disappears and the saddle bifurcation occurs, thus the nano-actuator drops onto the substrate. It is concluded that the damping effects increase the dynamic pull-in voltage of system because higher initial energy are required in order to cause dynamic instability of the nano-actuator.

Fig.12 shows the coupling influences of nonlocal stress and strain gradient parameters on time history and phase portrait of the nano-actuator at the dynamic pull-in state. It is seen from Fig.12(a) that as the nonlocal stress gradient parameter increases, the dynamic pull-in time increases while the dynamic pull-in deflection of system decreases. On the other hand, as the nonlocal strain gradient parameter increases, the dynamic pull-in time decreases while the dynamic pull-in deflection increases. Fig.12(b) shows that the maximum velocity of system decreases with the increase of nonlocal stress gradient parameter, while increases with the increment of strain gradient parameter. The coupling influences of nonlocal stress and strain 
gradient capture the significant size-dependence of dynamic pull-in instability by unifying stress and strain gradients from different physical characteristics of nanoscale materials and structures.

\section{Conclusion}

An analytical model of FGCNTs reinforced nano-actuator with damping effect, subjected to electrostatic loads are presented to study the dynamic pull-in instability, and the size effects of electrostatically actuated nano-device are characterized by coupling impacts of nonlocal elasticity and strain gradient theory in this work. Main conclusions are given by

1) The frequency of nano-actuator decreases with the increment of initial amplitude of the nano-actuator and applied voltage, then the frequency drops to zero at dynamic pull-in instability.

2) The frequency of nano-actuator decreases with the increase of nonlocal stress gradient parameter while increases with the increment of nonlocal strain gradient parameter.

3) The frequency of FGX-CNTRC type system is higher than that of UD-CNTRC and FGO-CNTRC due to the distinct stiffness enhancement of CNTs in nano-actuator.

4) The magnitude of non-dimensional deflection gradually declines due to the dissipative effects of damping and thus the dynamic pull-in voltage is larger than that of undamped system.

5) The maximum velocity of system decreases as the nonlocal stress gradient parameter increases whereas increase with the increment of strain gradient parameters.

\section{Appendix A}

The associated coefficients of the dimensionless nonlocal governing equation of motion are expressed as follows 


$$
\begin{aligned}
\psi_{i}= & \frac{H_{i+1}}{H_{0}}, i \in[0,5], \\
H_{0}= & K_{4} \int_{0}^{1} \phi \phi d \xi-\mu K_{4} \int_{0}^{1} \phi^{\prime \prime} \phi d \xi \\
H_{1}= & -\left[V_{e}^{2}(1+\gamma)+(1-n) \alpha_{3}+n \alpha_{4}\right] \int_{0}^{1} \phi d \xi \\
H_{2}= & \left(k_{3}-\mu k_{2} N^{T}\right) \int_{0}^{1} \phi^{(4)} \phi d \xi-\eta k_{3} \int_{0}^{1} \phi^{(6)} \phi d \xi+k_{2} N^{T} \int_{0}^{1} \phi^{\prime \prime} \phi d \xi \\
& -\left[V_{e}^{2}(2+\gamma)+3(1-n) \alpha_{3}+4 n \alpha_{4}\right] \int_{0}^{1} \phi\left(\phi-\mu \phi^{\prime \prime}\right) d \xi \\
H_{3}= & -\left[V_{e}^{2}(3+\gamma)+6(1-n) \alpha_{3}+10 n \alpha_{4}\right] \int_{0}^{1} \phi\left[\phi^{2}-2 \mu\left(\phi^{\prime}\right)^{2}-2 \mu \phi \phi^{\prime \prime}\right] d \xi \\
H_{4}= & k_{1}\left[\int_{0}^{1}\left(\phi^{\prime}\right)^{2} d \xi\right]\left(\mu \int_{0}^{1} \phi^{(4)} \phi d \xi-k_{1} \int_{0}^{1} \phi \phi^{\prime \prime} d \xi\right) \\
& -\left(V_{e}^{2}(4+\gamma)+10(1-n) \alpha_{3}+20 n \alpha_{4}\right) \int_{0}^{1} \phi^{2}\left[\phi^{2}-6 \mu\left(\phi^{\prime}\right)^{2}-3 \mu \phi \phi^{\prime \prime}\right] d \xi \\
H_{5}= & -\left(V_{e}^{2}(5+\gamma)+15(1-n) \alpha_{3}+35 n \alpha_{4}\right) \int_{0}^{1} \phi^{3}\left[\phi^{2}-12 \mu\left(\phi^{\prime}\right)^{2}-4 \mu \phi \phi^{\prime \prime}\right] d \xi \\
H_{6}= & \hat{C}_{s d}\left(\int_{0}^{1} \phi^{\prime} \phi d \xi-\mu \int_{0}^{1} \phi^{(3)} \phi d \xi\right)+\hat{C}_{v d}\left(\int_{0}^{1} \phi \phi d \xi-\mu \int_{0}^{1} \phi^{\prime \prime} \phi d \xi\right)
\end{aligned}
$$

\section{Acknowledge}

The authors acknowledge financial support from National Science Foundation of China under Number 11172165.

\section{References}

[1] T. Mousavi, S. Bornassi, H. Haddadpour, The effect of small scale on the pull-in instability of nano-switches using DQM, Int. J. Solids Struct. 50(2013) 1193-202.

[2] J. Chang, B.-K. Min, J. Kim, S.-J. Lee, L. Lin, Electrostatically actuated carbon nanowire nanotweezers, Smart Mater. Struct. 18(2009) 065017.

[3] M. Mojahedi, M. Ahmadian, K. Firoozbakhsh, The influence of the intermolecular surface forces on the static deflection and pull-in instability of the micro/nano cantilever gyroscopes, Compos. Part B 56(2014) 336-43.

[4] W.-M. Zhang, H. Yan, Z.-K. Peng, G. Meng, Electrostatic pull-in instability in MEMS/NEMS: A review, Sens. Actuators, A 214(2014) 187-218.

[5] A. Ramezani, A. Alasty, J. Akbari, Influence of van der Waals force on the pull-in parameters of cantilever type nanoscale electrostatic actuators, Microsyst. Technol. 12(2006) 1153-61.

[6] W.D. Yang, X. Wang, C.Q. Fang, G. Lu, Electromechanical coupling characteristics of carbon nanotube reinforced cantilever nano-actuator, Sens. Actuators, A 220(2014) 178-87.

[7] A.W. Rodriguez, F. Capasso, S.G. Johnson, The Casimir effect in microstructured geometries, Nat. 
Photonics 5(2011) 211-21.

[8] J. Zou, Z. Marcet, A.W. Rodriguez, M.T. Reid, A.P. McCauley, Kravchenko, II, et al., Casimir forces on a silicon micromechanical chip, Nat. Commun. 4(2013) 1845.

[9] R. Soroush, A. Koochi, A. Kazemi, A. Noghrehabadi, H. Haddadpour, M. Abadyan, Investigating the effect of Casimir and van der Waals attractions on the electrostatic pull-in instability of nano-actuators, Phys. Scr. 82(2010) 045801.

[10] A.C. Eringen, D. Edelen, On nonlocal elasticity, Int. J. Eng. Sci. 10(1972) 233-48.

[11] J.N. Reddy, Nonlocal theories for bending, buckling and vibration of beams, Int. J. Eng. Sci. 45(2007) 288-307.

[12] F. Yang, A. Chong, D. Lam, P. Tong, Couple stress based strain gradient theory for elasticity, Int. J. Solids Struct. 39(2002) 2731-43.

[13] S. Park, X. Gao, Bernoulli-Euler beam model based on a modified couple stress theory, J. Micromech. Microeng. 16(2006) 2355.

[14] H. Ma, X.-L. Gao, J. Reddy, A microstructure-dependent Timoshenko beam model based on a modified couple stress theory, J. Mech. Phys. Solids 56(2008) 3379-91.

[15] D. Lam, F. Yang, A. Chong, J. Wang, P. Tong, Experiments and theory in strain gradient elasticity, J. Mech. Phys. Solids 51(2003) 1477-508.

[16] B. Akgöz, Ö. Civalek, Strain gradient elasticity and modified couple stress models for buckling analysis of axially loaded micro-scaled beams, Int. J. Eng. Sci. 49(2011) 1268-80.

[17] B. Akgöz, Ö. Civalek, Longitudinal vibration analysis for microbars based on strain gradient elasticity theory, J. Vib. Control 20(2014) 606-16.

[18] C. Lim, G. Zhang, J. Reddy, A higher-order nonlocal elasticity and strain gradient theory and its applications in wave propagation, J. Mech. Phys. Solids 78(2015) 298-313.

[19] C. Polizzotto, A unifying variational framework for stress gradient and strain gradient elasticity theories, Eur. J. Mech. A-Solids 49(2015) 430-40.

[20] L. Li, Y. Hu, L. Ling, Flexural wave propagation in small-scaled functionally graded beams via a nonlocal strain gradient theory, Compos. Struct. 133(2015) 1079-92.

[21] L. Li, Y. Hu, L. Ling, Wave propagation in viscoelastic single-walled carbon nanotubes with surface effect under magnetic field based on nonlocal strain gradient theory, Physica E 75(2016) 118-24.

[22] M. Eltaher, M. Khater, S.A. Emam, A review on nonlocal elastic models for bending, buckling, vibrations, and wave propagation of nanoscale beams, Appl. Math. Model. (2015).

[23] J.N. Reddy, S. El-Borgi, J. Romanoff, Non-linear analysis of functionally graded microbeams using Eringen's non-local differential model, Int. J. Non Linear Mech. 67(2014) 308-18.

[24] J.N. Reddy, S. El-Borgi, Eringen's nonlocal theories of beams accounting for moderate rotations, Int. J. Eng. Sci. 82(2014) 159-77.

[25] Ö. Civalek, C. Demir, Buckling and bending analyses of cantilever carbon nanotubes using the euler-bernoulli beam theory based on non-local continuum model, Asian J. Civ. Eng. 12(2011) 651-61.

[26] K. Liew, Z. Lei, L. Zhang, Mechanical analysis of functionally graded carbon nanotube reinforced composites: A review, Compos. Struct. 120(2015) 90-7.

[27] B. Akgöz, Ö. Civalek, Thermo-mechanical buckling behavior of functionally graded microbeams embedded in elastic medium, Int. J. Eng. Sci. 85(2014) 90-104.

[28]H.-S. Shen, Nonlinear bending of functionally graded carbon nanotube-reinforced composite plates in thermal environments, Compos. Struct. 91(2009) 9-19.

[29] A. Alibeigloo, Static analysis of functionally graded carbon nanotube-reinforced composite plate 
embedded in piezoelectric layers by using theory of elasticity, Compos. Struct. 95(2013) 612-22.

[30] M.H. Yas, M. Heshmati, Dynamic analysis of functionally graded nanocomposite beams reinforced by randomly oriented carbon nanotube under the action of moving load, Appl. Math. Model. 36(2012) 1371-94.

[31] N. Wattanasakulpong, V. Ungbhakorn, Analytical solutions for bending, buckling and vibration responses of carbon nanotube-reinforced composite beams resting on elastic foundation, Comput. Mater. Sci. 71(2013) 201-8.

[32] H.-S. Shen, Thermal buckling and postbuckling behavior of functionally graded carbon nanotube-reinforced composite cylindrical shells, Compos. Part B 43(2012) 1030-8.

[33] H.-S. Shen, C.-L. Zhang, Thermal buckling and postbuckling behavior of functionally graded carbon nanotube-reinforced composite plates, Mater. \& Des. 31(2010) 3403-11.

[34] R. Ansari, T. Pourashraf, R. Gholami, A. Shahabodini, Analytical solution for nonlinear postbuckling of functionally graded carbon nanotube-reinforced composite shells with piezoelectric layers, Compos. Part B 90(2016) 267-77.

[35] M.H. Ghayesh, H. Farokhi, M. Amabili, Nonlinear behaviour of electrically actuated MEMS resonators, Int. J. Eng. Sci. 71(2013) 137-55.

[36] S. Azizi, M.-R. Ghazavi, G. Rezazadeh, S.E. Khadem, Thermo-elastic damping in a functionally graded piezoelectric micro-resonator, Int. J. Mech. Mater. Des. 11(2015) 357-69.

[37]C.-C. Liu, C.-C. Wang, Numerical investigation into nonlinear dynamic behavior of electrically-actuated clamped-clamped micro-beam with squeeze-film damping effect, Appl. Math. Model. 38(2014) 3269-80.

[38] M.I. Younis, F. Alsaleem, D. Jordy, The response of clamped-clamped microbeams under mechanical shock, Int. J. Non Linear Mech. 42(2007) 643-57.

[39] F. Tajaddodianfar, H.N. Pishkenari, M.R.H. Yazdi, E.M. Miandoab, Size-dependent bistability of an electrostatically actuated arch NEMS based on strain gradient theory, J. Phys. D: Appl. Phys. 48(2015) 245503.

[40] L.-L. Ke, J. Yang, S. Kitipornchai, Nonlinear free vibration of functionally graded carbon nanotube-reinforced composite beams, Compos. Struct. 92(2010) 676-83.

[41] M. Rafiee, J. Yang, S. Kitipornchai, Large amplitude vibration of carbon nanotube reinforced functionally graded composite beams with piezoelectric layers, Compos. Struct. 96(2013) 716-25.

[42] A. Ramezani, A. Alasty, J. Akbari, Closed-form solutions of the pull-in instability in nano-cantilevers under electrostatic and intermolecular surface forces, Int. J. Solids Struct. 44(2007) 4925-41.

[43] A. Ramezani, A. Alasty, Instability of nanocantilever arrays in electrostatic and van der Waals interactions, J. Phys. D: Appl. Phys. 42(2009) 225506.

[44] A. Ramezani, A. Alasty, J. Akbari, Pull-in parameters of cantilever type nanomechanical switches in presence of Casimir force, Nonlinear Anal.: Hybrid Systems 1(2007) 364-82.

[45] B. Wang, S. Zhou, J. Zhao, X. Chen, Size-dependent pull-in instability of electrostatically actuated microbeam-based MEMS, J. Micromech. Microeng. 21(2011) 027001.

[46] M. Mojahedi, M.T. Ahmadian, K. Firoozbakhsh, Dynamic Pull-in Instability and Vibration Analysis of a Nonlinear Microcantilever Gyroscope under Step Voltage Considering Squeeze Film Damping, Int. J. Appl. Mech. 05(2013) 1350032.

[47] L.-D. Liao, P.C. Chao, C.-W. Huang, C.-W. Chiu, DC dynamic and static pull-in predictions and analysis for electrostatically actuated clamped circular micro-plates based on a continuous model, J. Micromech. Microeng. 20(2010) 025013. 
[48] J.-H. He, Homotopy perturbation method for solving boundary value problems, Phys. Lett. A 350(2006) 87-8.

[49] J.-H. He, Homotopy perturbation method: a new nonlinear analytical technique, Appl. Math. and comput. 135(2003) 73-9.

[50] R.K. Gupta, E.S. Hung, Y.-J. Yang, G. Ananthasuresh, S.D. Senturia, Pull-in dynamics of electrostatically-actuated beams, Proc. Solid-State Sens. Actuator Workshop 1996.

[51] O. Bochobza-Degani, D. Elata, Y. Nemirovsky, An efficient DIPIE algorithm for CAD of electrostatically actuated MEMS devices, J. Microelectromech. Syst. 11(2002) 612-20.

[52] G.N. Nielson, G. Barbastathis, Dynamic pull-in of parallel-plate and torsional electrostatic MEMS actuators, J. Microelectromech. Syst.15(2006) 811-21.

[53] J. Yang, X.L. Jia, S. Kitipornchai, Pull-in instability of nano-switches using nonlocal elasticity theory, J. Phys. D: Appl. Phys. 41(2008) 035103.

[54] M. Baghani, Analytical study on size-dependent static pull-in voltage of microcantilevers using the modified couple stress theory, Int. J. Eng Sci. 54(2012) 99-105.

[55] Y. Okada, Y. Tokumaru, Precise determination of lattice parameter and thermal expansion coefficient of silicon between 300 and 1500 K, J. Appl. Phys. 56(1984) 314-20.

[56] C.-H. Cho, Characterization of Young' s modulus of silicon versus temperature using a "beam deflection" method with a four-point bending fixture, Curr. Appl Phys. 9(2009) 538-45.

[57] G.W. Vogl, A.H.Nayfeh, A reduced-order model for electrically actuated clamped circular plates. J. Micromech. Microeng. 15 (2005) 684-690. 


\section{Capital of Figures}

Fig.1. (a) Schema of FGCNTs reinforced nano-actuator,

(b) Geometrical distribution of CNTs in the matrix.

Fig. 2. Validation of time history by HPM and numerical method.

Fig.3. (a) Effects of nonlocal stress gradient parameter,

(b) Effects of strain gradient parameter,

where UD-CNTRC, $V_{c n t}=0.12, A=0.2, \Delta T=200 \mathrm{~K}, \gamma=0.65, \alpha_{4}=1$.

Fig.4. Effects of geometrical distribution of CNTs on the frequency of nano-actuator

for two nonlocal models, where (a) stress gradient model, (b) strain gradient model,

$$
V_{c n t}=0.12, V_{e}=5, A=0.2, \Delta T=200 \mathrm{~K}, \gamma=0.65, \alpha_{4}=1 \text {. }
$$

Fig.5. Effects of on volume fraction of CNTs on the frequency of FGX-CNTRC nano-actuator for two nonlocal models, where (a) stress gradient model,

(b) strain gradient model, and $V_{e}=5, A=0.2, \Delta T=200 \mathrm{~K}, \gamma=0.65, \alpha_{4}=1$.

Fig.6. Effects of temperature change on the frequency of FGX-CNTRC nano-actuator for two nonlocal models, where (a) stress gradient model, (b) strain gradient model,

$$
\text { and } V_{c n t}=0.12, V_{e}=5, A=0.2, \gamma=0.65, \alpha_{4}=1 \text {. }
$$

Fig.7. Effects of dispersion forces on the frequency of FGX-CNTRC actuator for two nonlocal models, where (a) stress gradient model, (b) strain gradient model,

$$
\text { and } V_{c n t}=0.12, V_{e}=5, A=0.2, \Delta T=200 \mathrm{~K}, \gamma=0.65 \text {. }
$$

Fig.8. Coupling effects of stress and strain gradient parameters on frequency of UD-CNTRC nano-actuator, where, $V_{c n t}=0.12, V_{e}=10, A=0.2$, $\Delta T=200 \mathrm{~K}, \gamma=0.65, \alpha_{4}=1$.

Fig.9. Effects of applied voltage on the frequency of high modes, where (a) nonlocal stress gradient parameter, (b) strain gradient parameter, and FGO-CNTRC, $V_{c n t}=0.12, A=0.2, \Delta T=200 \mathrm{~K}, \gamma=0.65, \alpha_{4}=1$. 
Fig. 10. The time history (a) and the phase portrait (b) of actuator near dynamic pull-in state, where $\mu=0.1$ and $\eta=0.1$, and the damping effects are taken as $\tilde{C}_{s d}=1$ and $\tilde{C}_{v d}=1$

Fig.11. The time history (a) and the phase portrait (b) of actuator near dynamic pull-in state, where $\mu=0.1$ and $\eta=0.1$, and without damping

$$
\text { system } \tilde{C}_{s d}=0 \text { and } \tilde{C}_{v d}=0 \text {. }
$$

Fig.12. Comparison of stress gradient and strain gradient parameters on the time history (a) and the phase portrait (b) of actuator with damping system at dynamic pull-in state.

Table 1. Referent parameters of dynamic pull-in experiment for verification of HPM.

Table 2. Comparison of dynamic pull-in voltage by different methods.

Table 3. Comparison of vibrational amplitudes by HPM and numerical method.

Table 4. Comparison of pull-in voltage with other size-dependent analytical models.

Table 5. Temperature-dependent material property of CNTs [28] and silicon $[55,56]$.

Table 6. CNTs efficient parameter of scale-dependent property for different volume fraction [40]. 


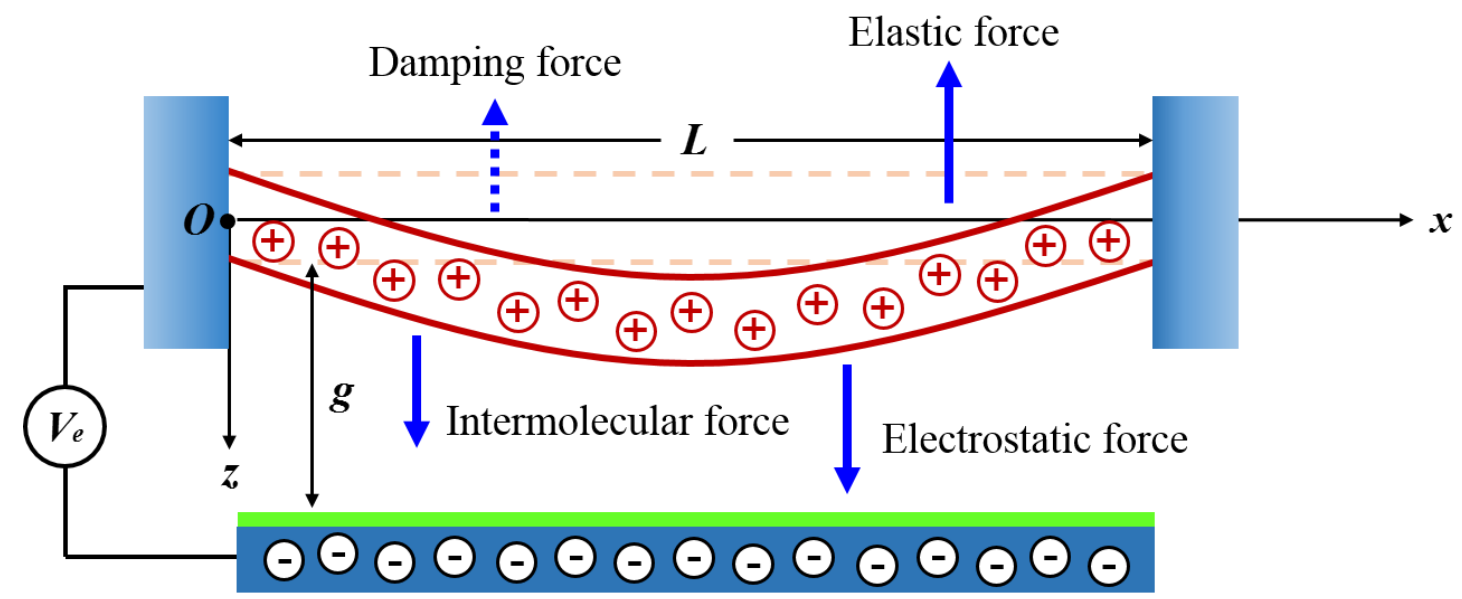

(a)

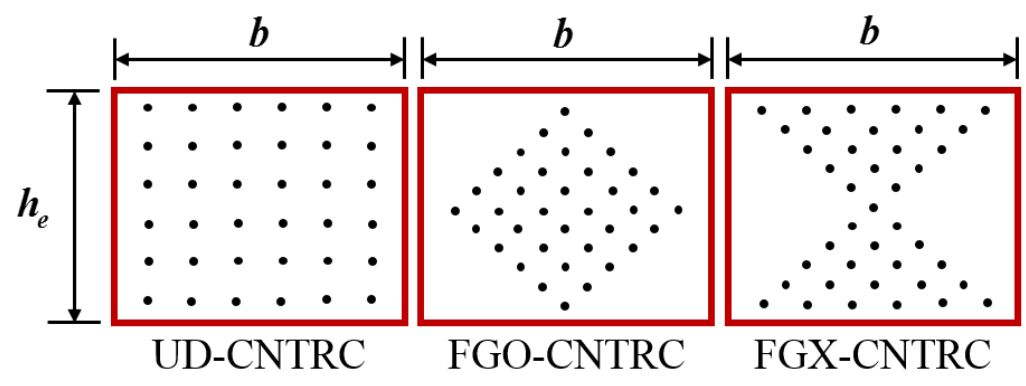

(b)

Fig.1. (a) Schema of FGCNTs reinforced nano-actuator;

(b) Geometrical distribution of CNTs in the matrix.

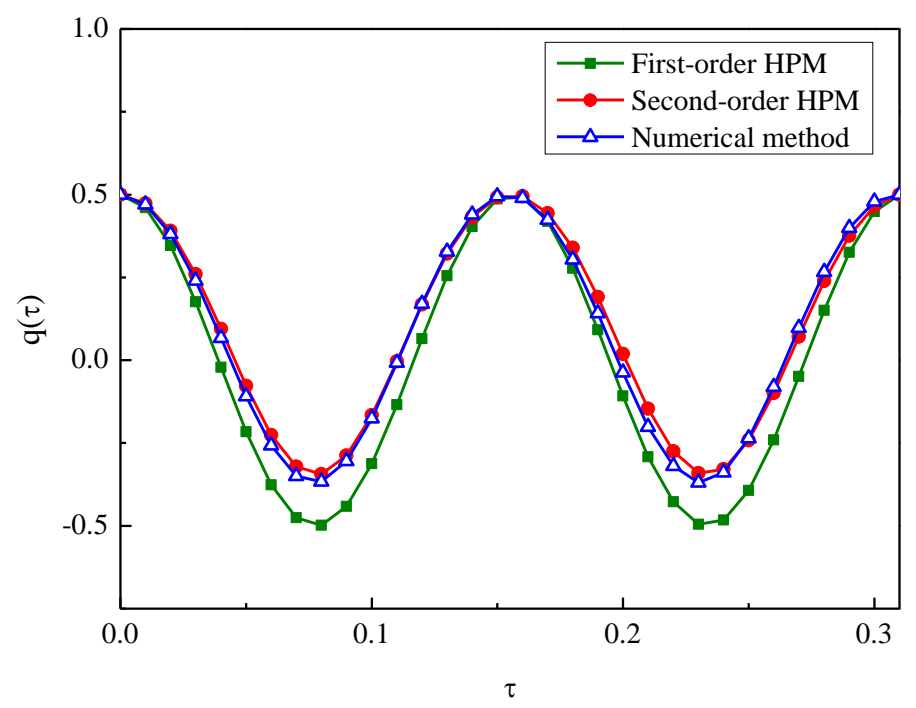

Fig. 2. Validation of time history by HPM and numerical method. 

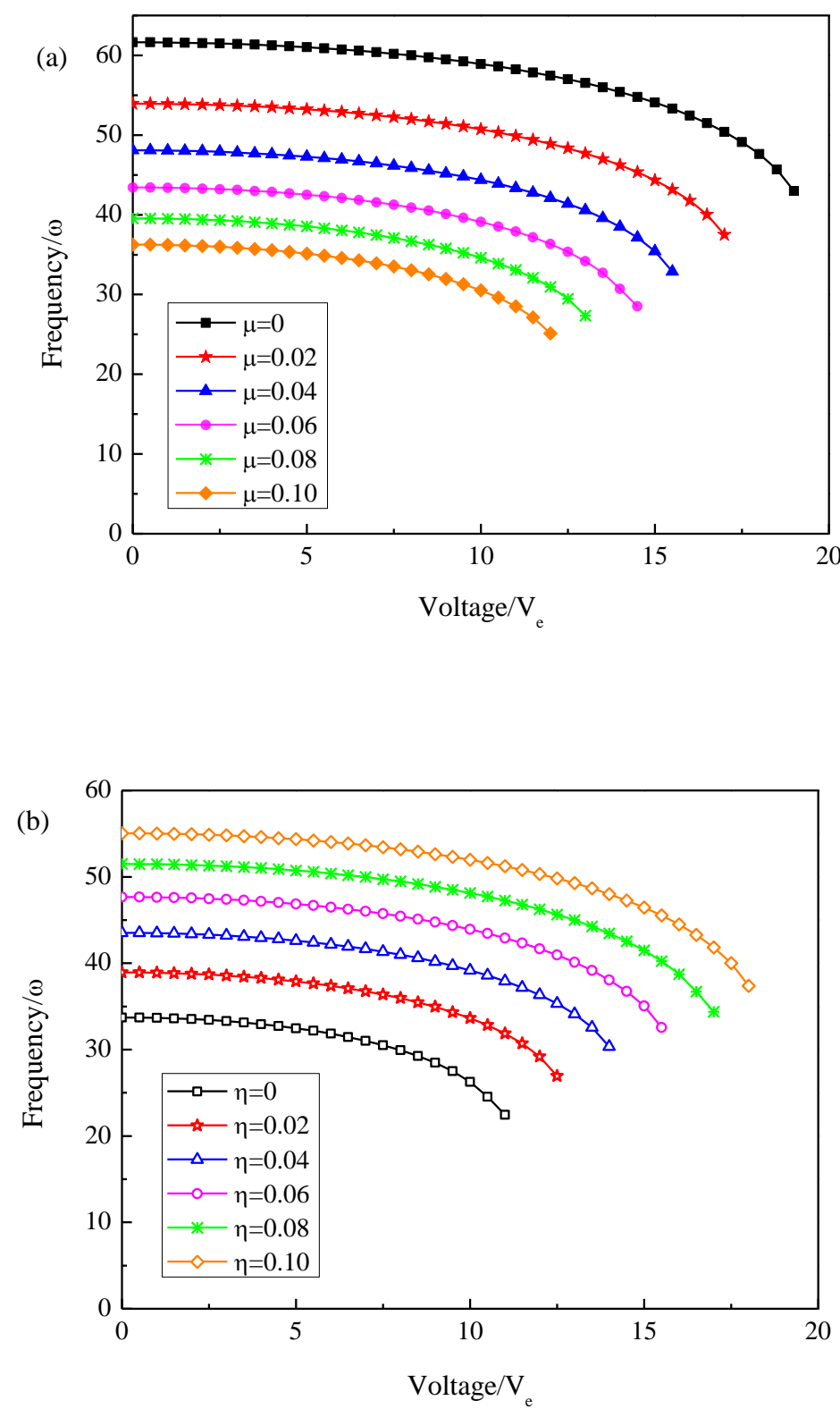

Fig.3. (a) Effects of nonlocal stress gradient parameter, (b) Effects of strain gradient parameter,

where UD-CNTRC, $V_{c n t}=0.12, A=0.2, \Delta T=200 \mathrm{~K}, \gamma=0.65, \alpha_{4}=1$. 

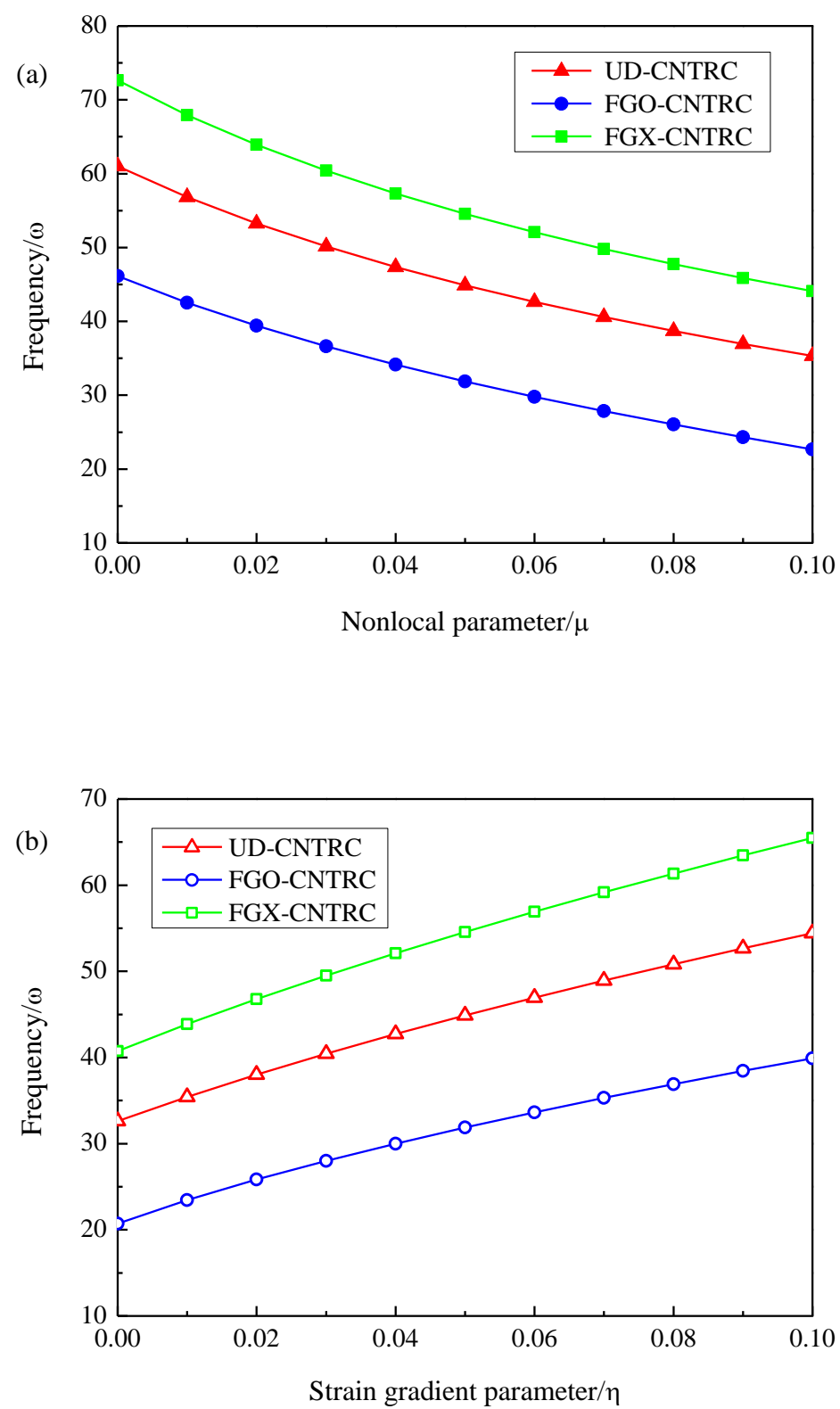

Fig.4. Effects of geometrical distribution of CNTs on the frequency of nano-actuator for two nonlocal models , where (a) stress gradient model, (b) strain gradient model,

$$
V_{c n t}=0.12, V_{e}=5, A=0.2, \Delta T=200 \mathrm{~K}, \gamma=0.65, \alpha_{4}=1 \text {. }
$$



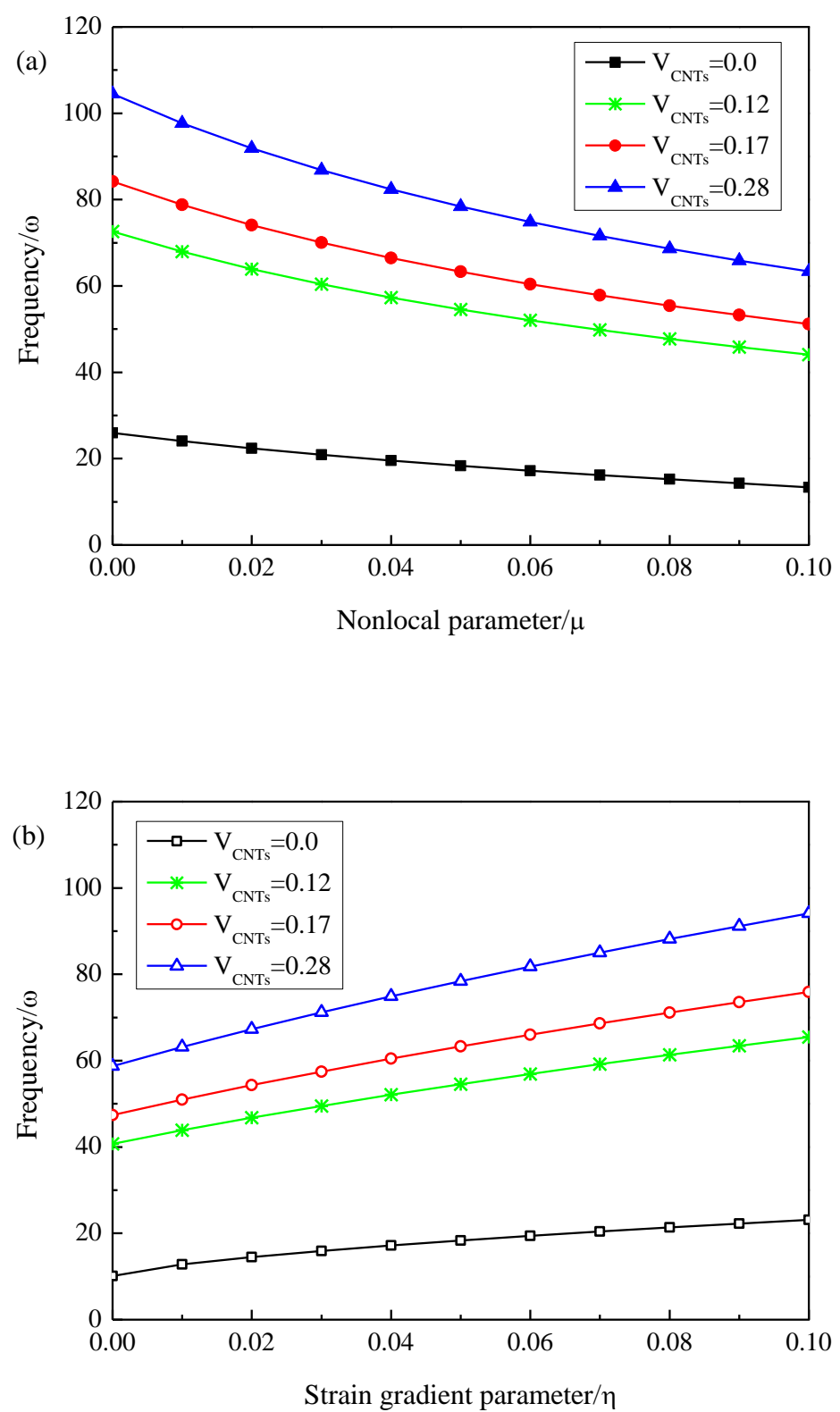

Fig.5. Effects of on volume fraction of CNTs on the frequency of FGX-CNTRC nano-actuator for two nonlocal models, where (a) stress gradient model,

(b) strain gradient model, and $V_{e}=5, A=0.2, \Delta T=200 \mathrm{~K}, \gamma=0.65, \alpha_{4}=1$. 

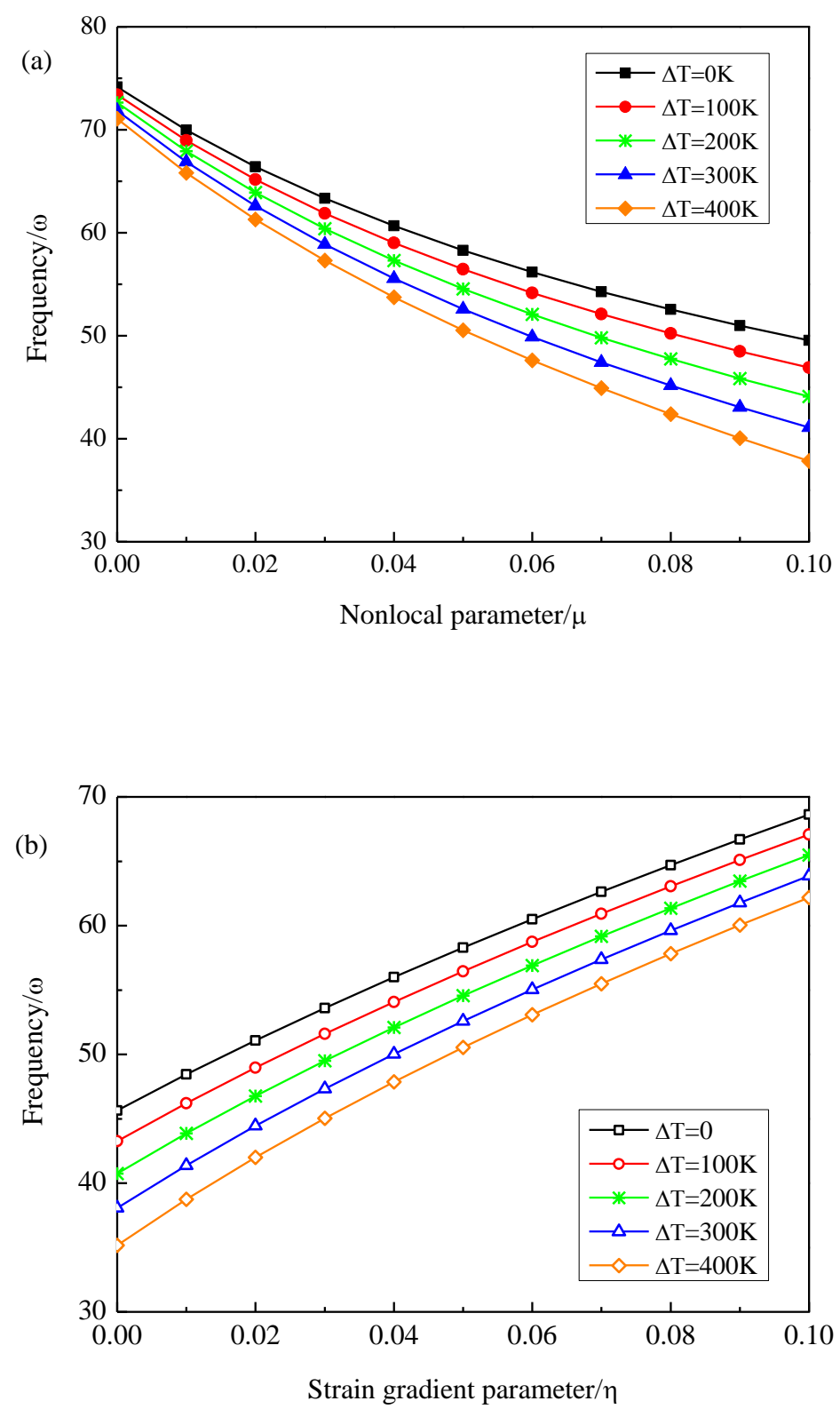

Fig.6. Effects of temperature change on the frequency of FGX-CNTRC nano-actuator for two nonlocal models, where (a) stress gradient model, (b) strain gradient model, and $V_{c n t}=0.12, V_{e}=5, A=0.2, \gamma=0.65, \alpha_{4}=1$. 

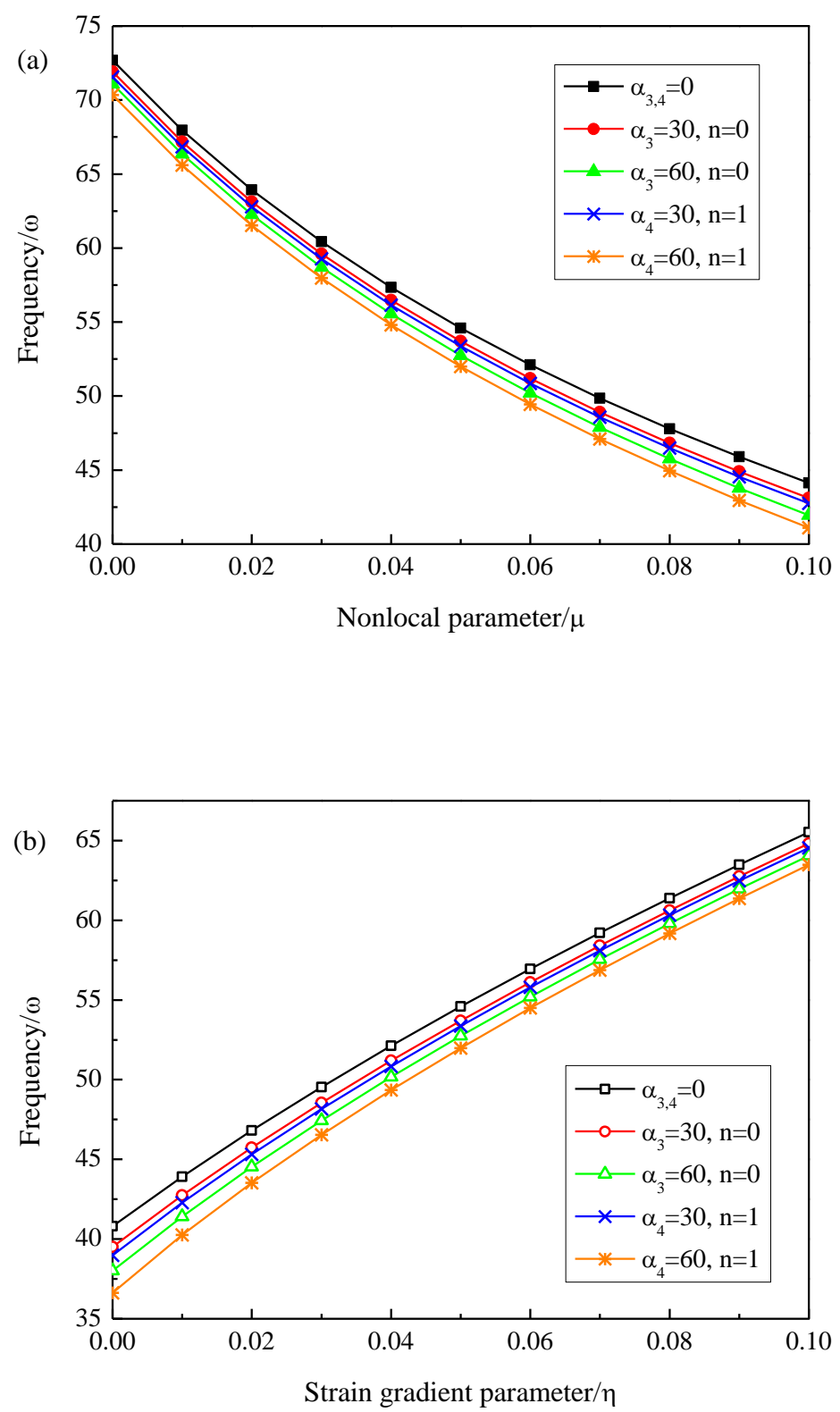

Fig.7. Effects of dispersion forces on the frequency of FGX-CNTRC actuator for two nonlocal models, where (a) stress gradient model, (b) strain gradient model, and $V_{c n t}=0.12, V_{e}=5, A=0.2, \Delta T=200 \mathrm{~K}, \gamma=0.65$. 


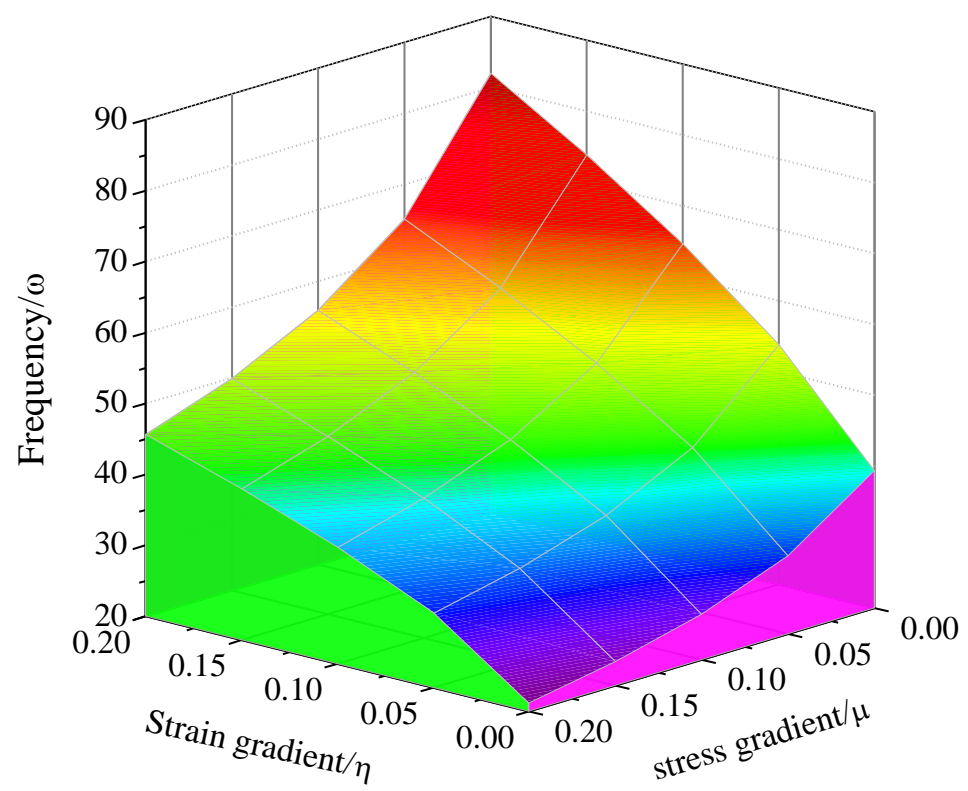

Fig.8.Coupling effects of stress and strain gradient parameters on frequency of UD-CNTRC nano-actuator, where $V_{c n t}=0.12, V_{e}=10, A=0.2$,

$$
\Delta T=200 \mathrm{~K}, \gamma=0.65, \alpha_{4}=1 \text {. }
$$

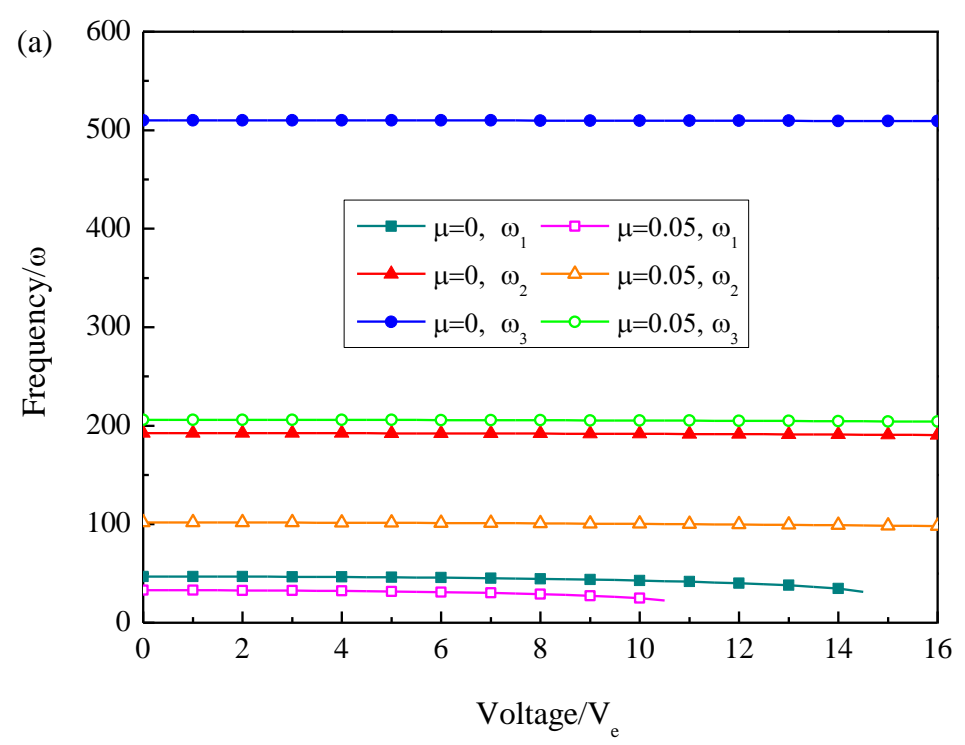




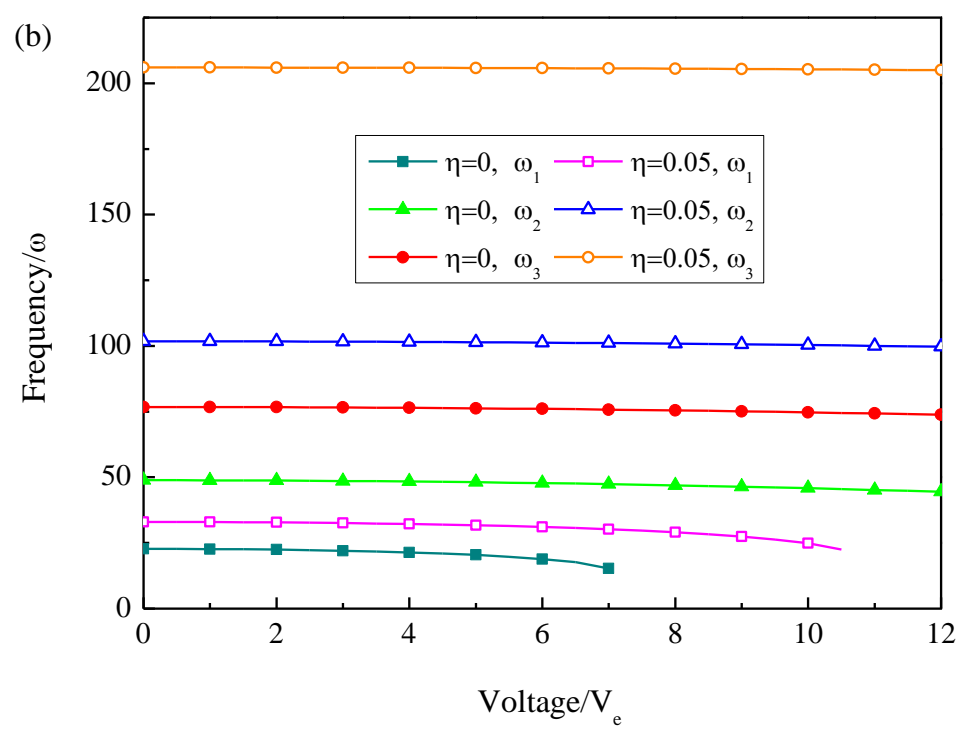

Fig.9. Effects of applied voltage on the frequency of high modes, where (a) nonlocal stress gradient parameter, (b) strain gradient parameter, and FGO-CNTRC, $V_{c n t}=0.12, A=0.2, \Delta T=200 \mathrm{~K}, \gamma=0.65, \alpha_{4}=1$.

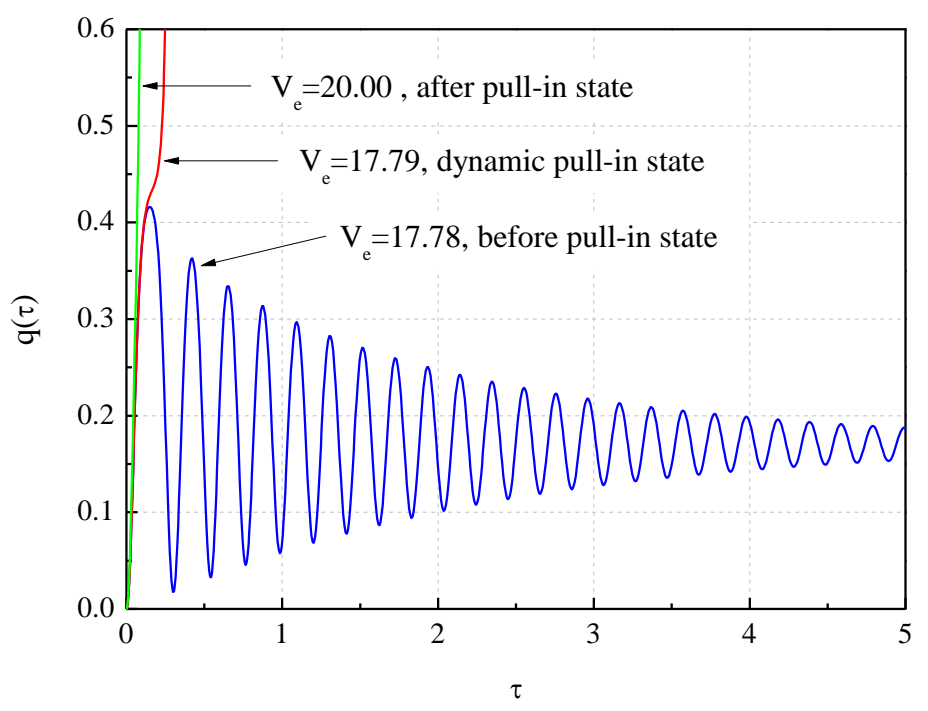

(a) 


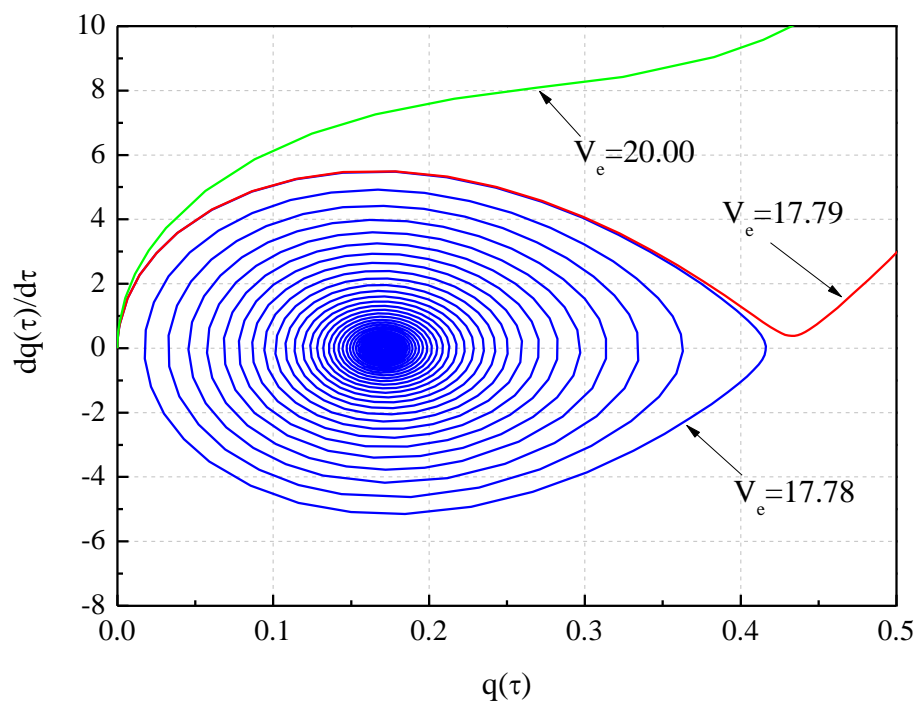

(b)

Fig. 10. The time history (a) and the phase portrait (b) of actuator near dynamic pull-in state, where $\mu=0.1$ and $\eta=0.1$, and the damping effects are taken as $\tilde{C}_{s d}=1$ and $\tilde{C}_{v d}=1$.

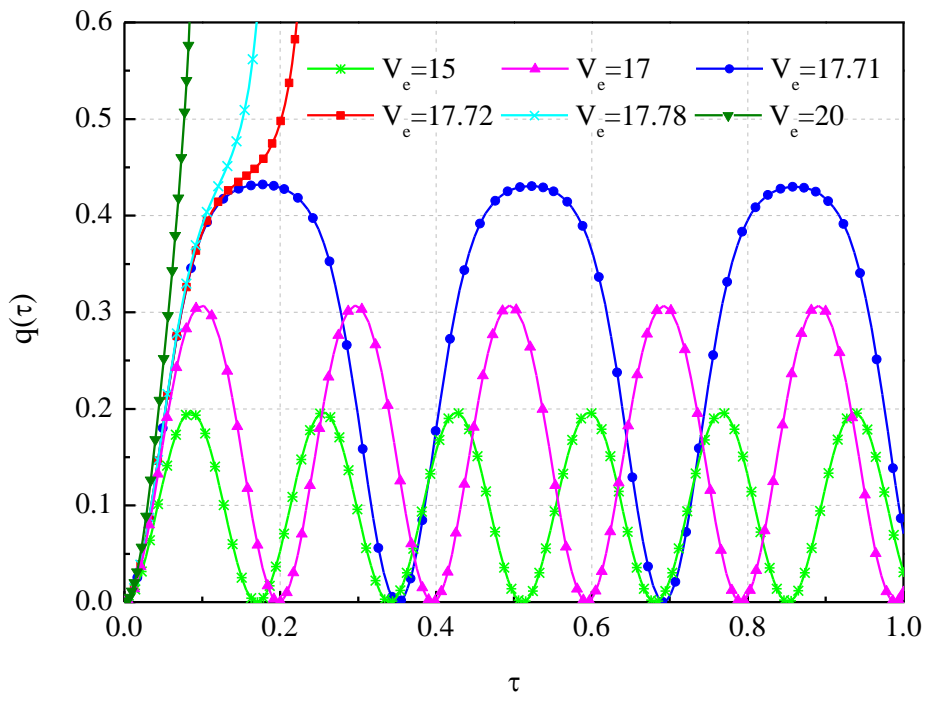

(a) 


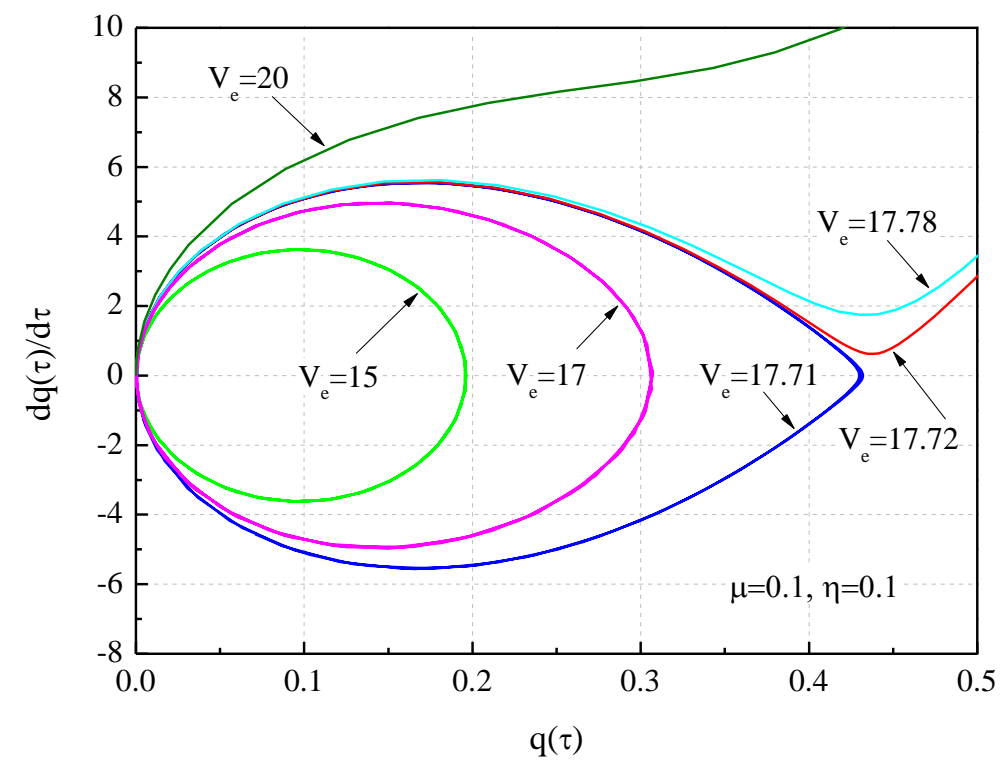

(b)

Fig.11. The time history (a) and the phase portrait (b) of actuator near dynamic pull-in state, where $\mu=0.1$ and $\eta=0.1$, and without damping

$$
\text { system } \tilde{C}_{s d}=0 \text { and } \tilde{C}_{v d}=0 \text {. }
$$

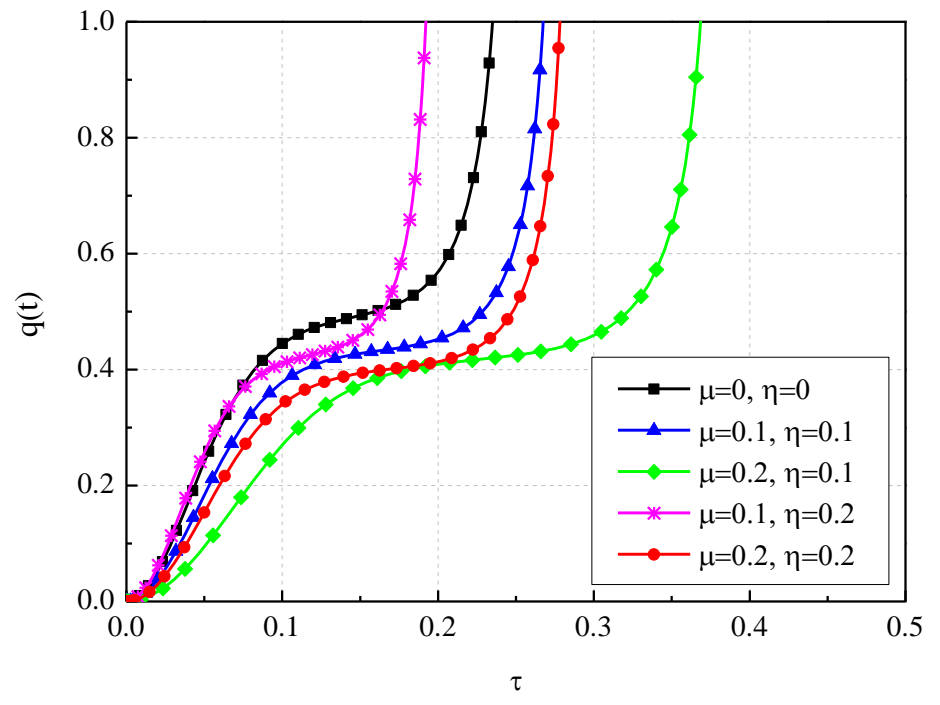

(a) 


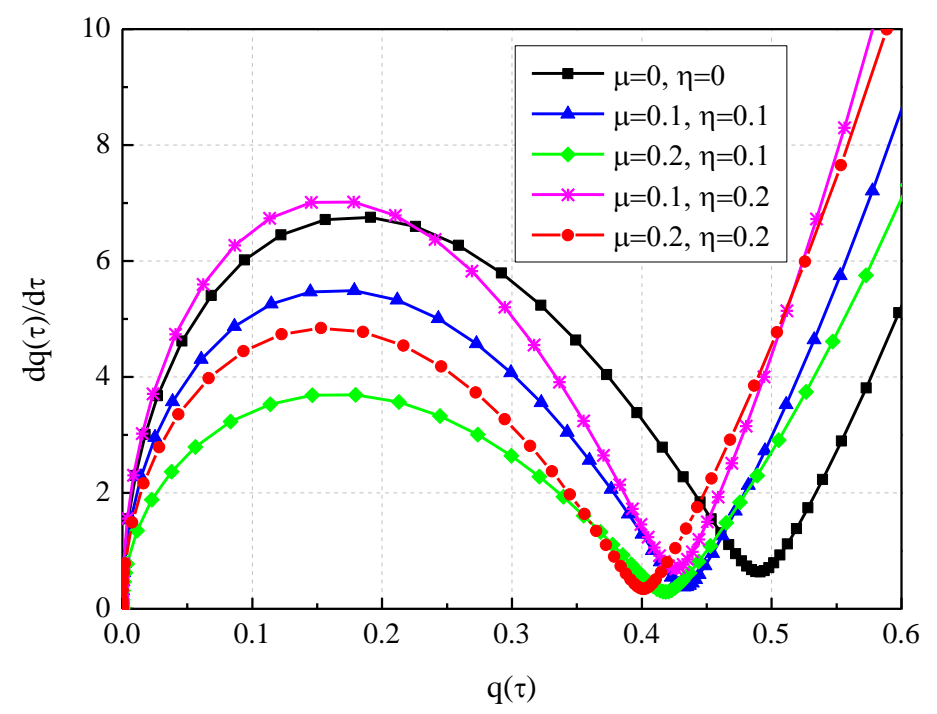

(b)

Fig. 12. Comparison of stress gradient and strain gradient parameters on the time history (a) and the phase portrait (b) of actuator with damping system at dynamic pull-in state.

Table 1

Referent parameters of dynamic pull-in experiment for verification of HPM.

\begin{tabular}{lll}
\hline Parameters & Symbol & Value/unit \\
\hline Length & $L$ & $610 \mu \mathrm{m}$ \\
Width & $b$ & $40 \mu \mathrm{m}$ \\
Height & $h$ & $2.12 \mu \mathrm{m}$ \\
Initial gap & $g_{0}$ & $2.07 \mu \mathrm{m}$ \\
Young's modulus & $E$ & $164 \mathrm{GPa}$ \\
Poisson's ratio & $v$ & 0.28 \\
Residual stress & $\sigma_{0}$ & $-3.5 \mathrm{MPa}$ \\
\hline
\end{tabular}


Table 2

Comparison of dynamic pull-in voltage by different methods.

\begin{tabular}{|c|c|c|c|}
\hline \multirow{2}{*}{ Method } & \multicolumn{2}{|c|}{ Dynamic pull-in voltage (V) } & \multirow{2}{*}{ Reference } \\
\hline & $\sigma_{0}=-3.5 \mathrm{MPa}$ & $\sigma_{0}=0$ & \\
\hline Experiment & 8.10 & - & {$[50]$} \\
\hline DIPIE algorithm & - & 10.752 & {$[51]$} \\
\hline EBM & 8.027 & 10.974 & {$[52]$} \\
\hline HPM & 7.985 & 11.108 & Present \\
\hline
\end{tabular}

Table 3

Comparison of vibrational amplitudes by HPM and numerical method.

\begin{tabular}{llllll}
\hline \multirow{2}{*}{$\begin{array}{l}\text { Initial } \\
\text { amplitude }\end{array}$} & \multicolumn{2}{l}{ Van der Waals force/ $\alpha_{3}$} & & \multicolumn{2}{l}{ Casimir force $\alpha_{4}$} \\
\cline { 2 - 3 } \cline { 5 - 6 } & Numerical & $2^{\text {nd }} \mathrm{HPM}$ & & Numerical & $2^{\text {nd }} \mathrm{HPM}$ \\
\hline 0 & 0.0039738 & 0.0039745 & & 0.0039450 & 0.0039457 \\
0.1 & 0.0950536 & 0.0951715 & & 0.0950612 & 0.0951844 \\
0.2 & 0.186546 & 0.186825 & & 0.186686 & 0.186986 \\
0.3 & 0.278728 & 0.279294 & & 0.279223 & 0.279872 \\
0.4 & 0.372013 & 0.373142 & & 0.373271 & 0.374671 \\
0.5 & 0.466961 & 0.469236 & & 0.469634 & 0.472728 \\
\hline
\end{tabular}


Table 4

Comparison of pull-in voltage with other size-dependent analytical models.

\begin{tabular}{llll}
\hline \multicolumn{1}{c}{ Model } & Method & Classical & Unclassical \\
\hline Nonlocal stress & LDL [53] & 1.616 & 0.452 \\
gradient theory & DQM [1] & 1.680 & 0.497 \\
$(\mu=0.1)$ & Present & 1.680 & 0.498 \\
Strain gradient & MVIM [54] & 9.87 & 10.10 \\
theory $(\eta=0.1)$ & Present & 9.853 & 10.082 \\
\hline
\end{tabular}

Table 5

Temperature-dependent material property of CNTs [28] and silicon $[55,56]$.

\begin{tabular}{lllll}
\hline Temperature $(\mathrm{K})$ & $E_{c n t}(\mathrm{TPa})$ & $\alpha_{c n t}\left(\times 10^{-6} / \mathrm{K}\right)$ & $E_{m}(\mathrm{MPa})$ & $\alpha_{m}\left(\times 10^{-6} / \mathrm{K}\right)$ \\
\hline 300 & 5.6466 & 3.4584 & 169.68 & 2.57 \\
400 & 5.5813 & 4.0919 & 167.69 & 3.14 \\
500 & 5.5308 & 4.5361 & 165.71 & 3.59 \\
600 & 5.4952 & 4.7438 & 163.72 & 3.88 \\
700 & 5.4744 & 4.6677 & 161.73 & 3.99 \\
\hline
\end{tabular}

Table 6

CNTs efficient parameter of scale-dependent property for different volume fraction [40].

\begin{tabular}{lllll}
\hline$V_{c n t}^{*}$ & 0 & 0.12 & 0.17 & 0.28 \\
$\eta$ & - & 1.2833 & 1.3414 & 1.3238 \\
\hline
\end{tabular}


A short biography of the author(s)

1) X. Wang a,

Professor of Engineering Mechanics, Master degree from Shanghai Jiaotong University in 1986. Current fields of interest: Smart structures

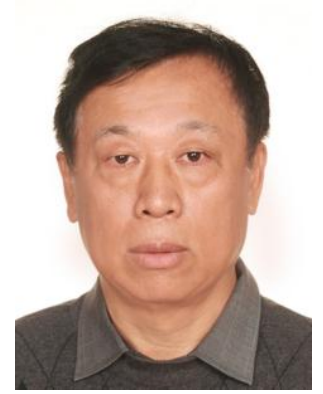

2) W.D.Yang a,

PhD graduate

Current fields of interest: Smart materials and structures

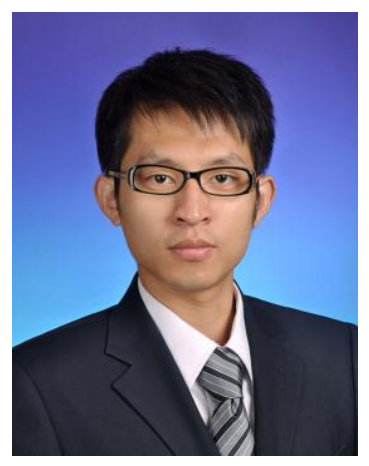

3) F.P.Yang a,

Associate Professor of Engineering Mechanics,, PhD from Shanghai Jiaotong University in 2005.

Current fields of interest: Composite materials and structures

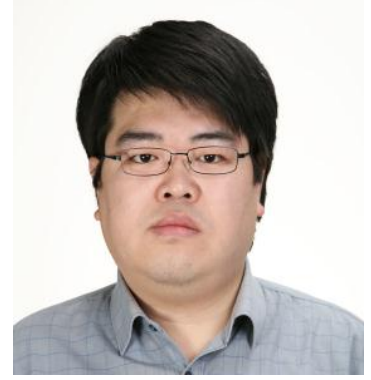

